\title{
Regio- and Enantioselective Pd-Catalyzed Allylic Alkylation of Ketones through Allyl Enol Carbonates
}

\author{
Barry M. Trost* and Jiayi Xu \\ Department of Chemistry, Stanford University, Stanford, California 94305-5080. \\ bmtrost@stanford.edu
}

\section{Experimental General}

All reactions were carried out under an atmosphere of nitrogen or argon in oven-dried glassware with magnetic stirring, unless otherwise indicated. Solvents were freshly purified by passage through an aluminum column before use. Solvents for Pd-catalyzed reactions were degassed by freeze-thaw techniques under vaccum ${ }^{1}$. Tris(dibenzylideneacetone)palladium(0) monochloroform complex, $\mathrm{Pd}_{2} \mathrm{dba}_{3} \cdot \mathrm{CHCl}_{3}$ was prepared by the procedure of Ibers ${ }^{2}$. Ligands $\mathbf{L 1}, \mathbf{L 2}, \mathbf{L 3}$, and $\mathbf{L} \mathbf{4}$ were prepared by literature procedures ${ }^{3,4}$. All other reagents were used as obtained unless otherwise noted.

Flash Chromatography was performed with EM Science silica gel (0.040-0.063 $\mu \mathrm{m}$ grade). Analytical thin-layer chromatography was performed with $0.2 \mathrm{~mm}$ coated commercial silica gel plates (E. Merck, DC-Plasrikfolien, kieselgel 60 F254). Melting points were obtained on a Thomas-Hoover apparatus in open capillary tubes and are uncorrected. Kugelrohr distillations were performed on a Büchi GKR-50 glass tube oven. Proton nuclear magnetic resonance $\left({ }^{1} \mathrm{H}-\mathrm{NMR}\right)$ data were acquired on a Mercury 400 (400 $\mathrm{MHz})$ or on a Varian Unity Inova-500 (500 MHz) spectrometer. Chemical shifts are reported in delta $(\delta)$ units, in parts per million (ppm) downfield from tetramethylsilane. Splitting patterns are designated as $\mathrm{s}$, singlet; d, doublet; t, triplet; q, quartet; p, pentet, m, multiplet, br, broad. Carbon-13 nuclear magnetic resonance $\left({ }^{13} \mathrm{C}\right.$-NMR) data were acquired at $100 \mathrm{MHz}$ on a Mercury 400 or at $125 \mathrm{MHz}$ on a Varian Unity Inova -500 spectrometer. Chemical shifts are reported in ppm relative to the center line of a triplet at 77.1 ppm for chloroform- $d$. Infrared (IR) data were recorded as films on sodium chloride plates or a potassium bromide $(\mathrm{KBr})$ pellets on a Perkin-Elmer Paragon 500 FT-IR spectrometer. Absorbance frequencies are reported in reciprocal centimeters $\left(\mathrm{cm}^{-1}\right)$. Elemental analyses (Anal.) were performed by M.-H.-W. Laboratories of Pheonix, AZ. Chiral GC analyses were performed on a HP 6890 series GC system using a Cyclosil B chiral column. GC analyses were performed on a HP 6850 series GC system using a Agilent Technologies HP-1 GC column (30 m length, $0.32 \mathrm{~mm}$ I.D., $0.25 \mu \mathrm{m}$ film). Chiral HPLC analyses were performed on a Themo Separation Products Spectra Series P-100 or 200 and UV100 (254 nm) using Chiralcel $^{\circledR}$ columns (OD, OB-H, OJ, AD, As, or OC) eluting with heptane / iso-propanol mixtures indicated. Optical rotations were measured on a Jasco DIP-1000 digital polarimeter using $5 \mathrm{~cm}$ cells and the sodium $\mathrm{D}$ line $(589 \mathrm{~nm})$ at ambient temperature in the solvent and concentration indicated.

The completed list of optimization studies of the Pd-catalyzed Asymmetric Allyl Alkylation of 2methyl cyclohexanone ${ }^{a}$.

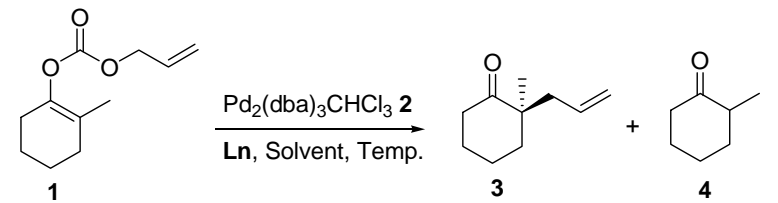

\begin{tabular}{|l|l|l|l|l|l|l|l|l|l|l|}
\hline Entry & Ln & Solvent & $\begin{array}{c}\text { Temp. } \\
{ }^{\circ} \mathrm{C}\end{array}$ & $\begin{array}{c}\text { Time } \\
\mathrm{h}\end{array}$ & $\mathrm{Pd} \%$ & $\mathbf{L n} \%$ & Additive $^{\text {ee }^{b} \%}$ & $\begin{array}{c}\text { Yield }^{c} \\
\text { \% of 3 }\end{array}$ & $\begin{array}{c}\text { Yield }^{c} \\
\text { \% of } \mathbf{4}\end{array}$ \\
\hline 1 & $\mathbf{L 1}$ & $\mathrm{CH}_{3} \mathrm{CN}$ & 23 & 20 & 5 & 5.5 & & NA & $<5$ & \\
\hline 2 & $\mathbf{L 2}$ & $\mathrm{CH}_{3} \mathrm{CN}$ & 23 & 20 & 5 & 5.5 & & NA & $<5$ & \\
\hline 3 & $\mathbf{L 3}$ & $\mathrm{CH}_{3} \mathrm{CN}$ & 23 & 20 & 5 & 5.5 & & NA & $<5$ & \\
\hline 4 & $\mathbf{L 4}$ & $\mathrm{CH}_{3} \mathrm{CN}$ & 23 & 20 & 5 & 5.5 & & NA & 0 & \\
\hline 5 & $\mathbf{L 2}$ & DCM & 23 & 20 & 5 & 5.5 & & 78 & 61 & 11 \\
\hline
\end{tabular}




\begin{tabular}{|c|c|c|c|c|c|c|c|c|c|c|}
\hline 6 & L3 & DCM & 23 & 20 & 5 & 5.5 & & 64 & 90 & 9 \\
\hline 7 & L4 & DCM & 23 & 20 & 5 & 5.5 & & 83 & 57 & 26 \\
\hline 8 & L4 & DCM & 23 & 20 & 5 & 5.5 & & 84 & 64 & 26 \\
\hline 9 & L4 & DCM & 4 & 20 & 5 & 5.5 & & 85 & 52 & 15 \\
\hline 10 & L4 & DCM & 23 & 20 & 5 & 5.5 & $\begin{array}{l}50 \% \\
\mathrm{Bu}_{4} \mathrm{NCl}\end{array}$ & 78 & 24 & 24 \\
\hline 11 & L4 & DCM & 23 & 20 & 5 & 5.5 & $\begin{array}{l}50 \% \\
\mathrm{Bu}_{4} \mathrm{NBr}\end{array}$ & 78 & 19 & 15 \\
\hline 12 & L4 & DCM & 23 & 20 & 5 & 5.5 & $\begin{array}{l}50 \% \\
\mathrm{Bu}_{4} \mathrm{NBF}_{4}\end{array}$ & 77 & 18 & 13 \\
\hline 13 & L4 & DCM & 23 & 20 & 5 & 5.5 & $\begin{array}{l}50 \% \\
\text { TBAT }\end{array}$ & NA & Trace & Trace \\
\hline 14 & L1 & Dioxane & 23 & 20 & 5 & 5.5 & & 49 & 87 & 3 \\
\hline 15 & L2 & Dioxane & 23 & 20 & 5 & 5.5 & & 74 & 68 & 0 \\
\hline 16 & L3 & Dioxane & 23 & 20 & 5 & 5.5 & & 66 & 92 & 3 \\
\hline 17 & L4 & Dioxane & 23 & 20 & 5 & 5.5 & & 80 & 99 & 0 \\
\hline 18 & L1 & DME & 23 & 4 & 5 & 7.5 & & 65 & $59 *$ & \\
\hline 19 & L2 & DME & 23 & 48 & 5 & 7.5 & & 52 & $35 *$ & \\
\hline 20 & L3 & DME & 23 & 4 & 5 & 7.5 & & 76 & $81 *$ & \\
\hline 21 & L4 & DME & 23 & 24 & 5 & 7.5 & & 85 & $60 *$ & \\
\hline 22 & L1 & DME & 23 & 5 & 5 & 5.5 & & 66 & 81 & 8 \\
\hline 23 & L2 & DME & 23 & 24 & 5 & 5.5 & & 72 & 48 & 1 \\
\hline 24 & L3 & DME & 23 & 4 & 5 & 5.5 & & 76 & 87 & 1.6 \\
\hline 25 & L4 & DME & 23 & 24 & 5 & 5.5 & & 84 & 87 & 7 \\
\hline 26 & L4 & DME & 23 & 3 & 5 & 12.5 & & 84 & 77 & 0.7 \\
\hline 27 & L4 & $\mathrm{DME}$ & 4 & 24 & 5 & 5.5 & & 87 & 69 & 19 \\
\hline 28 & L4 & $\begin{array}{l}\mathrm{DME} \\
1 \% \mathrm{H}_{2} \mathrm{O}\end{array}$ & 23 & 24 & 5 & 5.5 & & NA & 20 & 3.7 \\
\hline 29 & L3 & $\begin{array}{l}\mathrm{DME} \\
1 \% \mathrm{H}_{2} \mathrm{O}\end{array}$ & 23 & 24 & 5 & 5.5 & & NA & 1.5 & 0 \\
\hline 30 & L1 & DMSO & 23 & 40 & 5 & 5.5 & & NA & 0 & \\
\hline 31 & L2 & DMSO & 23 & 40 & 5 & 5.5 & & NA & 0 & \\
\hline 32 & L3 & DMSO & 23 & 40 & 5 & 5.5 & & 68 & 55 & 15.5 \\
\hline 33 & L4 & DMSO & 23 & 40 & 5 & 5.5 & & NA & 0 & \\
\hline 34 & L2 & THF & 23 & 12 & 5 & 5.5 & & 75 & 80 & 2 \\
\hline 35 & L3 & $\mathrm{THF}$ & 23 & 12 & 5 & 5.5 & & 72 & 89 & 1 \\
\hline
\end{tabular}




\begin{tabular}{|l|l|l|l|l|l|l|l|l|l|l|}
\hline 36 & L4 & THF & 23 & 12 & 5 & 5.5 & & 81 & 85 & 1 \\
\hline 37 & L1 & Toluene & 23 & 2 & 5 & 5.5 & & 31 & 73 & 0 \\
\hline 38 & $\mathbf{L 2}$ & Toluene & 23 & 16 & 5 & 5.5 & & 61 & 73 & 2 \\
\hline 39 & L3 & Toluene & 23 & 16 & 5 & 5.5 & & 60 & 85 & 1 \\
\hline 40 & L4 & Toluene & 23 & $16(2)$ & 5 & 5.5 & & 85 & 88 & 0 \\
\hline 41 & L4 & Toluene & 4 & 16 & 5 & 5.5 & & 87 & 91 & 2 \\
\hline 42 & $\mathbf{L 4}$ & Toluene & $4(.3 \mathrm{M})$ & 16 & 5 & 5.5 & & 85 & 94 & 4 \\
\hline 43 & $\mathbf{L 4}$ & Toluene & -10 & 20 & 5 & 5.5 & & 83 & 84 & 6 \\
\hline 44 & $\mathbf{L 4}$ & Toluene & $4(.3 \mathrm{M})$ & 16 & 5 & 12.5 & & 86 & 88 & 4 \\
\hline 45 & $\mathbf{L 4}$ & Toluene & $\begin{array}{l}4 \\
(.025 \mathrm{M})\end{array}$ & 16 & 5 & 5.5 & & 87 & 92 & 0 \\
\hline 46 & $\mathbf{L 4}$ & Toluene & $4(.3 \mathrm{M})$ & 24 & 2 & 5 & & 85 & 88 & 5 \\
\hline
\end{tabular}

${ }^{a}$ Unless otherwise indicated, all reactions were performed on a $0.3 \mathrm{mmol}$ scale at $0.1 \mathrm{M}$; DCM stands for dichloromethane, DME stands for 1, 2-dimethoxylethane, THF stands for tetrahydrofuran. ${ }^{b}$ The ee values were determined by chiral GC. ${ }^{c}$ The yields were determined by quantitative GC analysis using decane as internal reference. ${ }^{*}$ The yields were isolated yields.

\section{Gereral Procedure for the Pd-catalyzed Reaction of Carbonates.}

The catalyst is sensitive to air and water and thus thorough degassing of the solvent needs to be performed. Two test tubes were connected with a double-end needle. One test tube was loaded with $2.5 \mathrm{~mol} \%$ $\mathrm{Pd}_{2}(\mathrm{dba})_{3} \mathrm{CHCl}_{3}$ (2) and $5.5 \mathrm{~mol} \% \mathrm{mmol}$ ligand; the other one was loaded with $0.30 \mathrm{mmol}$ carbonate. The system was evacuated and flushed with Argon three times at which point $1.5 \mathrm{ml}$ degassed solvent was added to both of the test tubes. After stirred for $20 \mathrm{~min}$ the orange solution of catalyst was transferred into the test tube containing carbonate substrate. In most cases, the color of the reaction solution turned to light yellow within a few minutes and then turned back to orange in different time depending on the choice of ligand, substrate, solvent and temperature, indicating the completion of the reaction. For GC analysis, the reaction mixture was diluted with $3 \mathrm{ml} \mathrm{10 \%}$ diethyl ether in petroleum ether and passed through a $3 \mathrm{~cm}$ layer of silica gel in a normal glass pipette. Otherwise, the reaction mixture was concentrated in vacuo and purified by column chromatography on silica gel eluted with 5\% diethyl ether in petroleum ether.<smiles>C=CCC1CCCCC1=O</smiles>

(R)-(+)-2-Allyl-2-methylcyclohexanone (3) ${ }^{5}$ : Colorless oil. $\mathrm{R}_{\mathrm{f}}=0.34$ (Diethyl ether/petroleum ether 1:9); $[\alpha]_{\mathrm{D}}^{22}=+49.6\left(\mathrm{c}=2.9, \mathrm{CH}_{2} \mathrm{Cl}_{2}, 85 \%\right.$ ee); $\mathrm{GC}$ (Cyclosil B column, $90{ }^{\circ} \mathrm{C}$ constant, 50:1 split ratio, 15.0 split flow, 1.3 flow rate; $\mathrm{t}_{1}=32.86 \mathrm{~min}$ (minor), $\mathrm{t}_{2}=34.95 \mathrm{~min}$ (major) $) ;{ }^{1} \mathrm{H} \mathrm{NMR}\left(400 \mathrm{MHz}, \mathrm{CDCl}_{3}\right): \delta=5.70$ (m, 1H), 5.08-5.01 (m, 2H), $2.38(\mathrm{~m}, 3 \mathrm{H}), 2.23$ (dd, $\left.J_{1}=14 \mathrm{~Hz}, J_{2}=7.6 \mathrm{~Hz}, 1 \mathrm{H}\right), 1.90-1.65$ (m, 5H), $1.62-$ 1.54 (m, 1H), 1.07 (s, 3H). ${ }^{13} \mathrm{C}$ NMR (100 MHz, $\left.\mathrm{CDCl}_{3}\right): \delta=215.6,133.9,118.0,48.5,42.0$, 38.9, 38.7, 27.5, 22.7, 21.1.<smiles>C=CCC1CCc2ccccc2C1=O</smiles>

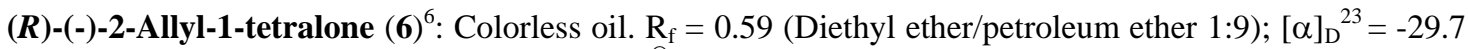
(c = 1.21, MeOH, 97\% ee); HPLC (Chiralcel ${ }^{\circledR}$ OD column; 2000:1 Heptane / Isopropanol; flow rate $=1$ $\mathrm{mL} / \mathrm{min} ; \mathrm{t}_{1}=21.87$ min (minor), $\mathrm{t}_{2}=23.88 \min \left(\right.$ major)); IR (film): $\tilde{\nu}_{\max }=3073(\mathrm{w}), 2930$ (m), $2862(\mathrm{w})$, 1682 (s), 1640 (w), 1600 (s), 1455 (m), 1434 (m), 1359 (w), 1280 (m), 1220 (s), 1156 (m), 996 (m), 911 
(m), $745 \mathrm{~cm}^{-1}(\mathrm{~s}) ;{ }^{1} \mathrm{H}$ NMR (400 MHz, $\left.\mathrm{CDCl}_{3}\right): \delta=8.03(\mathrm{~d}, J=8.0 \mathrm{~Hz}, 1 \mathrm{H}), 7.45\left(\mathrm{td}, J_{1}=7.4 \mathrm{~Hz}, J_{2}=1.2\right.$ Hz, 1H), 7.30 (t, $J=7.6 \mathrm{~Hz}, 1 \mathrm{H}), 7.23$ (d, $J=7.6 \mathrm{~Hz}, 1 \mathrm{H}), 5.85$ (m, 1H), 5.08 (m, 2H), 2.99 (dd, $J_{1}=7.6$ $\left.\mathrm{Hz}, J_{2}=4.4 \mathrm{~Hz}, 2 \mathrm{H}\right), 2.76(\mathrm{~m}, 1 \mathrm{H}), 2.55(\mathrm{~m}, 1 \mathrm{H}), 2.25(\mathrm{~m}, 2 \mathrm{H}), 1.86(\mathrm{~m}, 1 \mathrm{H}) ;{ }^{13} \mathrm{C} \mathrm{NMR}\left(100 \mathrm{MHz}, \mathrm{CDCl}_{3}\right)$ : $\delta=199.5,144.1,136.3,133.3,132.6,128.8,127.5,126.6,116.9,47.2,34.1,28.7,28.0$.<smiles>C=CCC1(CC=C)CCc2ccccc2C1=O</smiles>

2, 2-Diallyl-1-tetralone (8) ${ }^{7}$ : Colorless oil. $\mathrm{R}_{\mathrm{f}}=0.65$ (Diethyl ether/petroleum ether 1:9); ${ }^{1} \mathrm{H}$ NMR (300 MHz, $\left.\mathrm{CDCl}_{3}\right): \delta=8.03(\mathrm{~d}, J=8.0 \mathrm{~Hz}, 1 \mathrm{H}), 7.45(\mathrm{t}, J=7.4 \mathrm{~Hz}, 1 \mathrm{H}), 7.23(\mathrm{~m}, 2 \mathrm{H}), 5.85(\mathrm{~m}, 2 \mathrm{H}), 5.08(\mathrm{~m}$, $4 \mathrm{H}), 2.99(\mathrm{t}, J=6 \mathrm{~Hz}, 2 \mathrm{H}), 2.55\left(\mathrm{dd}, J_{1}=12.0 \mathrm{~Hz}, J_{2}=7.6 \mathrm{~Hz}, 2 \mathrm{H}\right), 2.25\left(\mathrm{dd}, J_{1}=12.0 \mathrm{~Hz}, J_{2}=7.6 \mathrm{~Hz}\right.$, $2 \mathrm{H}), 2.03(\mathrm{t}, J=6 \mathrm{~Hz}, 2 \mathrm{H})$.<smiles>C=CCC1CCCCC1=O</smiles>

(R)-(+)-2-Allylcyclohexanone (10) ${ }^{8}$ : Colorless oil. $\mathrm{R}_{\mathrm{f}}=0.35$ (Diethyl ether/petroleum ether 1:9); $[\alpha]_{\mathrm{D}}{ }^{24}=$ +10.28 (c = 1.1, $\mathrm{MeOH}, 78 \%$ ee); ${ }^{1} \mathrm{H}$ NMR (400 MHz, $\left.\mathrm{CDCl}_{3}\right): \delta=5.77(\mathrm{~m}, 1 \mathrm{H}), 5.08-4.80(\mathrm{~m}, 2 \mathrm{H}), 2.55$ (m, 1H), 2.34 (m, 3H), $2.18-1.94$ (m, 3H), $1.86(\mathrm{~m}, 1 \mathrm{H}), 1.66(\mathrm{~m}, 2 \mathrm{H}), 1.36(\mathrm{~m}, 1 \mathrm{H})$.

Determination of the ee value of $\mathbf{1 0}$ :

By our Chiral GC columns we had a hard time to separate the enantiomers of 10. Therefore, $\mathbf{1 0}$ was reduced to alcohol 32 and 33 by $\mathrm{NaBH}_{4}$ in $\mathrm{MeOH}$ in 1:2 ratio. 32 and $\mathbf{3 3}$ were separated by column chromatography on silica gel, eluted with $10 \% \mathrm{Et}_{2} \mathrm{O}$ in petroleum ether. 32 was dissolved in pyridine and treated with benzoyl chloride at room temperature. The reaction mixture was stirred for $2 \mathrm{~h}$ and most solvent was removed in vacuo. The residue was purified by column chromatography on silica gel eluted with $5 \% \mathrm{Et}_{2} \mathrm{O}$ in petroleum ether to yield 34. Compound $\mathbf{3 4}$ was ready to be separated on chiral GC (Cyclosil B column, $150{ }^{\circ} \mathrm{C}$ constant, 50:1 split ratio, 15.0 split flow, 1.5 flow rate; $\mathrm{t}_{1}=50.48 \mathrm{~min}$ (minor), $\mathrm{t}_{2}=51.54 \min ($ major)).<smiles>C=CCC1CCCCC1=O</smiles>

9<smiles>C=CCC1CCCCC1O</smiles>

32
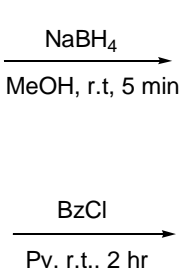

Py, r.t., 2 hr

Py, r.t, 2 hr<smiles>C=CCC1CCCCC1O</smiles>

33<smiles>C=CCC1CCCCC1OCC</smiles>

34<smiles>C=CC[C@H]1CCc2ccccc2C1=O</smiles>

(R)-(+)-2-Allyl-2-methyl-1-tetralone $(\mathbf{1 2})^{9}$ : Colorless oil. $\mathrm{R}_{\mathrm{f}}=0.60$ (Diethyl ether/petroleum ether 1:9); $[\alpha]_{\mathrm{D}}^{23}=+16.9\left(\mathrm{c}=1.39, \mathrm{CH}_{2} \mathrm{Cl}_{2}, 100 \%\right.$ ee); HPLC (Chiralcel ${ }^{\circledR}$ OB-H column; 97:3 Heptane / Isopropanol; flow rate $=1 \mathrm{~mL} / \mathrm{min} ; \mathrm{t}_{1}=5.79 \mathrm{~min}$ (major), $\mathrm{t}_{2}=6.74 \mathrm{~min}(\operatorname{minor})$ ); IR (film): $\tilde{\nu}_{\max }=3074(\mathrm{w}), 2966(\mathrm{~m})$, 2930 (s), 2856 (w), 1682 (s), 1640 (w), 1602 (m), 1455 (m), 1433 (w), 1375 (w), 1323 (w), 1287 (w), 1221 (s), 1156 (w), 1097 (w), $996(w), 972(w), 917(\mathrm{~m}), 742 \mathrm{~cm}^{-1}(\mathrm{~s}) ;{ }^{1} \mathrm{H}$ NMR (400 MHz, $\left.\mathrm{CDCl}_{3}\right): \delta=8.04$ (d, $J=8.0 \mathrm{~Hz}, 1 \mathrm{H}), 7.45\left(\mathrm{td}, J_{1}=7.4 \mathrm{~Hz}, J_{2}=1.2 \mathrm{~Hz}, 1 \mathrm{H}\right), 7.30(\mathrm{t}, J=7.6 \mathrm{~Hz}, 1 \mathrm{H}), 7.23(\mathrm{~d}, J=7.6 \mathrm{~Hz}, 1 \mathrm{H})$, $5.79(\mathrm{~m}, 1 \mathrm{H}), 5.08(\mathrm{~d}, J=10.8 \mathrm{~Hz}, 1 \mathrm{H}), 5.06(\mathrm{~d}, J=5.6 \mathrm{~Hz}, 1 \mathrm{H}), 2.98(\mathrm{~m}, 2 \mathrm{H}), 2.46\left(\mathrm{dd}, J_{1}=13.6 \mathrm{~Hz}, J_{2}=\right.$ $7.2 \mathrm{~Hz}, 1 \mathrm{H}), 2.27\left(\mathrm{dd}, J_{1}=13.6 \mathrm{~Hz}, J_{2}=7.6 \mathrm{~Hz}, 1 \mathrm{H}\right), 2.08(\mathrm{~m}, 1 \mathrm{H}), 1.90(\mathrm{~m}, 1 \mathrm{H}), 1.19(\mathrm{~s}, 3 \mathrm{H}) ;{ }^{13} \mathrm{C} \mathrm{NMR}$ (100 MHz, $\left.\mathrm{CDCl}_{3}\right): \delta=202.2,143.6,134.2,133.3,131.8,128.9,128.2,126.9,118.4,44.8,41.3$, 33.6, 25.6, 22.1. 


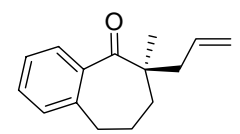

(-)-2-Allyl-2-methyl-1-benzosuberone (14) ${ }^{10}$ : Colorless oil. $\mathrm{R}_{\mathrm{f}}=0.49$ (Diethyl ether/petroleum ether 1:9); $[\alpha]_{\mathrm{D}}{ }^{24}=-6.93\left(\mathrm{c}=1.47, \mathrm{CH}_{2} \mathrm{Cl}_{2}, 91 \%\right.$ ee); GC (Cyclosil B ${ }^{\circledR}$ column; $130{ }^{\circ} \mathrm{C}$ constant temperature; $50: 1$ split ratio; 15.0 split flow; 1.5 flow rate; $\mathrm{t}_{1}=72.75 \mathrm{~min}$ (minor), $\mathrm{t}_{2}=74.49 \mathrm{~min}$ (major)); IR (film): $\tilde{\nu}_{\max }=$ 3073 (w), 2933 (s), 2864 (m), 1682 (s), 1640 (w), 1600 (m), 1482 (w), 1453 (m), 1375 (w), 1300 (w), 1251 (m), $1192(\mathrm{w}), 1095(\mathrm{w}), 997(\mathrm{w}), 964(\mathrm{~m}), 916(\mathrm{~m}), 786(\mathrm{w}), 759(\mathrm{~m}), 743 \mathrm{~cm}^{-1}(\mathrm{~m}) ;{ }^{1} \mathrm{H}$ NMR $(400 \mathrm{MHz}$, $\left.\mathrm{CDCl}_{3}\right): \delta=7.34(\mathrm{~m}, 1 \mathrm{H}), 7.25(\mathrm{~d}, J=8.0 \mathrm{~Hz}, 2 \mathrm{H}), 7.10(\mathrm{t}, J=7.6 \mathrm{~Hz}, 1 \mathrm{H}), 5.72(\mathrm{~m}, 1 \mathrm{H}), 5.06-5,00(\mathrm{~m}$, 2H), 2.77 (m, 2H), $2.32(\mathrm{~m}, 2 \mathrm{H}), 1.91$ (p, $J=6.8 \mathrm{~Hz}, 2 \mathrm{H}), 1.75(\mathrm{~m}, 1 \mathrm{H}), 1.61(\mathrm{~m}, 1 \mathrm{H}), 1.17(\mathrm{~s}, 3 \mathrm{H}) ;{ }^{13} \mathrm{C}$ NMR (100 MHz, $\left.\mathrm{CDCl}_{3}\right): \delta=214.5,141.6,137.3,133.7,130.8,128.4,127.2,126.6,118.4,49.2$, 43.9, 34.6, 32.9, 22.8, 22.3.

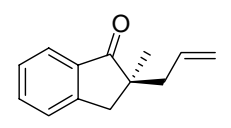

(+)-2-Allyl-2-methylindanone (16): Colorless oil. $\mathrm{R}_{\mathrm{f}}=0.43$ (Diethyl ether/petroleum ether 1:9); $[\alpha]_{\mathrm{D}}{ }^{22}=$ +46.2 (c = 1.6, $\mathrm{CH}_{2} \mathrm{Cl}_{2}, 76 \%$ ee); HPLC (Chiralcel ${ }^{\circledR}$ OB-H column; 2000:1 Heptane / Isopropanol; flow rate $=1 \mathrm{~mL} / \mathrm{min} ; \mathrm{t}_{1}=9.49 \min \left(\right.$ major), $\mathrm{t}_{2}=12.62 \mathrm{~min}$ (minor)); IR (film): $\tilde{\nu}_{\max }=3076(\mathrm{w}), 2962(\mathrm{~m})$, 2926 (m), 2867 (w), 1715 (s), 1640 (w), 1609 (s), 1588 (w), 1465 (m), 1435 (m), 1372 (w), 1330 (w), 1296 (m), 1205 (w), 1152 (w), $1091(\mathrm{w}), 983$ (m), 919 (m), 796 (m), $740 \mathrm{~cm}^{-1}$ (s); ${ }^{1} \mathrm{H} \mathrm{NMR}\left(400 \mathrm{MHz}, \mathrm{CDCl}_{3}\right)$ : $\delta=7.75(\mathrm{~d}, J=7.6 \mathrm{~Hz}, 1 \mathrm{H}), 7.59$ (t, $J=7.6 \mathrm{~Hz}, 1 \mathrm{H}), 7.43$ (d, $J=7.6 \mathrm{~Hz}, 1 \mathrm{H}), 7.37$ (t, $J=7.6 \mathrm{~Hz}, 1 \mathrm{H}), 5.66$ (m, 1H), $5.12-4.98(\mathrm{~m}, 2 \mathrm{H}), 3.17(\mathrm{~d}, J=17.2 \mathrm{~Hz}, 1 \mathrm{H}), 2.84(\mathrm{~d}, J=17.2 \mathrm{~Hz}, 1 \mathrm{H}), 2.39$ (dd, $J_{1}=13.6 \mathrm{~Hz}$, $\left.J_{2}=6.4 \mathrm{~Hz}, 1 \mathrm{H}\right), 2.30\left(\mathrm{dd}, J_{1}=8.2 \mathrm{~Hz}, J_{2}=6.4 \mathrm{~Hz}, 1 \mathrm{H}\right), 1.23(\mathrm{~s}, 3 \mathrm{H}) ;{ }^{13} \mathrm{C} \mathrm{NMR}\left(100 \mathrm{MHz}, \mathrm{CDCl}_{3}\right): \delta=$ 210.9, 152.7, 135.9, 135.0, 133.9, 127.5, 126.7, 124.3, 118.4, 48.9, 42.6, 39.4, 23.9; Anal. Calcd. for $\mathrm{C}_{13} \mathrm{H}_{14} \mathrm{O}$ : C, 83.83; H, 7.58; Found: C, 84.16; H, 7.61.

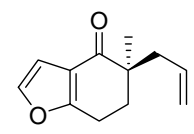

(+)-5-Allyl-5-methyl-6,7-dihydrobenzofuran-4(5H)-one $(\mathbf{1 8})^{10}$ : Colorless oil. $\mathrm{R}_{\mathrm{f}}=0.22$ (Diethyl ether/petroleum ether 1:9); $[\alpha]_{\mathrm{D}}^{23}=+8.6\left(\mathrm{c}=1.4, \mathrm{CH}_{2} \mathrm{Cl}_{2}, 82 \%\right.$ ee); HPLC (Chiralcel ${ }^{\circledR}$ AD column; 99.5:0.5 Heptane / Isopropanol; flow rate $=0.8 \mathrm{~mL} / \mathrm{min} ; \mathrm{t}_{1}=18.14 \mathrm{~min}$ (minor), $\mathrm{t}_{2}=20.70$ min (major)); IR (film): $\tilde{\nu}_{\max }=3150(\mathrm{w}), 3074(\mathrm{w}), 2966(\mathrm{w}), 2933(\mathrm{~m}), 2856(\mathrm{w}), 1677(\mathrm{~s}), 1640(\mathrm{w}), 1603(\mathrm{w}), 1456$ (m), 1440 (w), 1378 (w), 1356 (w), $1289(\mathrm{w}), 1253$ (w), 1219 (w), $1121(\mathrm{~m}), 1024(\mathrm{w}), 999(\mathrm{w}), 939(\mathrm{w})$, $918(\mathrm{w}), 739 \mathrm{~cm}^{-1}(\mathrm{w}) ;{ }^{1} \mathrm{H}$ NMR $\left(400 \mathrm{MHz}, \mathrm{CDCl}_{3}\right): \delta=7.31(\mathrm{~d}, J=2.0 \mathrm{~Hz}, 1 \mathrm{H}), 6.66(\mathrm{~d}, J=2.0 \mathrm{~Hz}, 1 \mathrm{H})$, $5.77(\mathrm{~m}, 1 \mathrm{H}), 5.12-5.03(\mathrm{~m}, 2 \mathrm{H}), 2.88(\mathrm{t}, J=6.4 \mathrm{~Hz}, 2 \mathrm{H}), 2.42\left(\mathrm{dd}, J_{1}=14.0 \mathrm{~Hz}, J_{2}=7.2 \mathrm{~Hz}, 1 \mathrm{H}\right), 2.24$ (dd, $\left.J_{1}=14 \mathrm{~Hz}, J_{2}=7.6 \mathrm{~Hz}, 1 \mathrm{H}\right), 2.12(\mathrm{~m}, 1 \mathrm{H}), 1.92(\mathrm{~m}, 1 \mathrm{H}), 1.15(\mathrm{~s}, 3 \mathrm{H}) ;{ }^{13} \mathrm{C} \mathrm{NMR}\left(100 \mathrm{MHz}, \mathrm{CDCl}_{3}\right): \delta$ = 198.5, 165.4, 143.0, 134.1, 119.8, 118.3, 107.3, 45.2, 41.2, 33.4, 21.9, 20.7 .<smiles>C=CCC1CCCC(=Cc2ccccc2)C1=O</smiles>

(+)-2-Allyl-6-benzylidene-2-methylcyclohexanone $\quad(\mathbf{2 0})^{9}$ : $\quad$ Colorless oil. $\quad \mathrm{R}_{\mathrm{f}}=0.50$ (Diethyl ether/petroleum ether 1:9); $[\alpha]_{\mathrm{D}}{ }^{24}=+22.9\left(\mathrm{c}=2.75, \mathrm{CH}_{2} \mathrm{Cl}_{2}, 95 \%\right.$ ee); HPLC (Chiralcel ${ }^{\circledR}$ OJ column; 97:3 Heptane / Isopropanol; flow rate $=1 \mathrm{~mL} / \mathrm{min} ; \mathrm{t}_{1}=6.43 \mathrm{~min}$ (minor), $\mathrm{t}_{2}=7.68 \mathrm{~min}$ (major)); IR (film): $\tilde{\nu}$ $\max _{\text {max }} 3074(\mathrm{w}), 3024(\mathrm{w}), 2966(\mathrm{w}), 2933$ (s), $2870(\mathrm{~m}), 1679(\mathrm{~s}), 1639(\mathrm{w}), 1595$ (s), $1573(\mathrm{~m}), 1492(\mathrm{~m})$, 1446 (m), 1373 (w), 1317 (w), 1265 (m), 1145 (s), 1113 (w), 1030 (w), 996 (m), 917 (m), 857 (w), $762(\mathrm{~m})$, $695 \mathrm{~cm}^{-1}(\mathrm{~s}) ;{ }^{1} \mathrm{H}$ NMR (400 MHz, $\left.\mathrm{CDCl}_{3}\right): \delta=7.37(\mathrm{~m}, 5 \mathrm{H}), 5.75(\mathrm{~m}, 1 \mathrm{H}), 5.07(\mathrm{~m}, 2 \mathrm{H}), 2.82(\mathrm{~m}, 2 \mathrm{H})$, $2.43\left(\mathrm{dd}, J_{1}=14.0 \mathrm{~Hz}, J_{2}=7.2 \mathrm{~Hz}, 1 \mathrm{H}\right), 2.23\left(\mathrm{dd}, J_{1}=14 \mathrm{~Hz}, J_{2}=7.6 \mathrm{~Hz}, 1 \mathrm{H}\right), 1.91(\mathrm{~m}, 1 \mathrm{H}), 1.73(\mathrm{~m}, 3 \mathrm{H})$, 1.15 (s, 3H); ${ }^{13} \mathrm{C}$ NMR (100 MHz, $\left.\mathrm{CDCl}_{3}\right): \delta=206.9,137.0,136.0,135.6,134.0,130.2,128.4,128.3$, 118.2, 47.1, 42.9, 35.1, 29.5, 24.2, 19.8 
<smiles>C=CCC1CSc2ccccc2C1=O</smiles>

(-)-3-Allyl-2,3-dihydrothiochromen-4-one (22) ${ }^{11}$ : Colorless oil. $\mathrm{R}_{\mathrm{f}}=0.47$ (Diethyl ether/petroleum ether 1:9); $[\alpha]_{\mathrm{D}}^{23}=-98.83$ (c = 1.27, $\mathrm{CH}_{2} \mathrm{Cl}_{2}, 93 \%$ ee); HPLC (Chiralcel ${ }^{\circledR}$ OB-H column; 99.5:0.5 Heptane / Isopropanol; flow rate $=1 \mathrm{~mL} / \mathrm{min} ; \mathrm{t}_{1}=10.68 \mathrm{~min}$ (major), $\mathrm{t}_{2}=11.87 \mathrm{~min}$ (minor)); IR (film): $\tilde{\boldsymbol{\nu}}_{\max }=$ 3075 (w), 3024 (w), 2917 (m), 2849 (w), 1682 (s), 1640 (w), 1588 (s), 1573 (m), 1459 (m), 1436 (s), 1342 (w), 1289 (m), $1211(\mathrm{~m}), 1162(\mathrm{w}), 1118(\mathrm{w}), 1087(\mathrm{w}), 997(\mathrm{w}), 919(\mathrm{~m}), 763(\mathrm{~m}), 736,695 \mathrm{~cm}^{-1}(\mathrm{~m}) ;{ }^{1} \mathrm{H}$ NMR (400 MHz, $\left.\mathrm{CDCl}_{3}\right): \delta=8.09(\mathrm{~m}, 1 \mathrm{H}), 7.36(\mathrm{~m}, 1 \mathrm{H}), 7.24(\mathrm{~m}, 1 \mathrm{H}), 7.16(\mathrm{~m}, 1 \mathrm{H}), 5.81(\mathrm{~m}, 1 \mathrm{H}), 5.14$ (m, 2H), 3.25 (dd, $\left.J_{1}=13.2 \mathrm{~Hz}, J_{2}=4.0 \mathrm{~Hz}, 1 \mathrm{H}\right), 3.08$ (dd, $\left.J_{1}=13.2 \mathrm{~Hz}, J_{2}=10.0 \mathrm{~Hz}, 1 \mathrm{H}\right), 2.84(\mathrm{~m}, 1 \mathrm{H})$, $2.73(\mathrm{~m}, 1 \mathrm{H}), 2.39(\mathrm{~m}, 1 \mathrm{H}) ;{ }^{13} \mathrm{C}$ NMR $\left(100 \mathrm{MHz}, \mathrm{CDCl}_{3}\right): \delta=195.5,141.8,135.0,133.1,130.6,129.6$, 127.4, 124.9, 118.1, 46.5, 33.1, 30.3.<smiles>C=CCC1CCc2cc(OC)ccc2C1=O</smiles>

(-)-2-Allyl-6-methoxy-3,4-dihydronaphthalen-1(2H)-one (24): White solid, mp $=35-36{ }^{\circ} \mathrm{C} . \mathrm{R}_{\mathrm{f}}=0.24$ (Diethyl ether/petroleum ether 1:9); $[\alpha]_{\mathrm{D}}{ }^{24}=-55.88$ (c $=3.8, \mathrm{CH}_{2} \mathrm{Cl}_{2}, 100 \%$ ee); HPLC (Chiralcel ${ }^{\circledR}$ OD column; 2000:1 Heptane / Isopropanol; flow rate $=1 \mathrm{~mL} / \mathrm{min} ; \mathrm{t}_{1}=48.72 \mathrm{~min}$ (minor), $\mathrm{t}_{2}=53.24 \mathrm{~min}$ (major)); IR (film): $\tilde{\nu}_{\max }=3075$ (w), 3024 (w), 2917 (m), 2849 (w), 1674 (s), 1640 (w), 1601 (s), 1573 (m), 1494 (m), 1454 (m), 1359 (m), 1335 (m), 1251 (s), 1226 (m), 1155 (w), 1133 (m), 1029 (m), 913 (m), 851 (w), $830 \mathrm{~cm}^{-1}(\mathrm{w}) ;{ }^{1} \mathrm{H}$ NMR $\left(400 \mathrm{MHz}, \mathrm{CDCl}_{3}\right): \delta=8.01$ (d, $\left.J=8.8 \mathrm{~Hz}, 1 \mathrm{H}\right), 6.81$ (dd, $J_{1}=8.8 \mathrm{~Hz}, J_{2}=2.4$ $\mathrm{Hz}, 1 \mathrm{H}), 6.68(\mathrm{~d}, J=2.4 \mathrm{~Hz}, 1 \mathrm{H}), 5.84(\mathrm{~m}, 1 \mathrm{H}), 5.14-5.04(\mathrm{~m}, 2 \mathrm{H}), 3.85(\mathrm{~s}, 3 \mathrm{H}), 2.94$ (dd, $J_{1}=7.6 \mathrm{~Hz}, J_{2}$ $=4.8 \mathrm{~Hz}, 2 \mathrm{H}), 2.75(\mathrm{~m}, 1 \mathrm{H}), 2.49(\mathrm{~m}, 1 \mathrm{H}), 2.23(\mathrm{~m}, 2 \mathrm{H}), 1.84(\mathrm{~m}, 1 \mathrm{H}) ;{ }^{13} \mathrm{C} \mathrm{NMR}\left(100 \mathrm{MHz}, \mathrm{CDCl}_{3}\right): \delta=$ 198.3, 163.4, 146.6, 136.5, 129.9, 126.2, 116.8, 113.2, 112.5, 55.4, 46.8, 34.2, 29.0, 28.0; Anal. Calcd. for $\mathrm{C}_{14} \mathrm{H}_{16} \mathrm{O}_{2}$ : C, 77.75; H, 7.46; Found: C, 78.12 ; H, 7.60.<smiles>C=CCC1CCc2ccc(OC)cc2C1=O</smiles>

(-)-2-Allyl-7-methoxy-3,4-dihydronaphthalen-1(2H)-one (26): Colorless oil. $\mathrm{R}_{\mathrm{f}}=0.33$ (Diethyl ether/petroleum ether 1:9); $[\alpha]_{\mathrm{D}}{ }^{24}=-50.2\left(\mathrm{c}=2.1, \mathrm{CH}_{2} \mathrm{Cl}_{2}, 97 \%\right.$ ee); HPLC (Chiralcel ${ }^{\circledR}$ OC column; $^{2}$ 99.5:0.5 Heptane / Isopropanol; flow rate $=1 \mathrm{~mL} / \mathrm{min} ; \mathrm{t}_{1}=25.23 \mathrm{~min}$ (major), $\mathrm{t}_{2}=30.86 \mathrm{~min}$ (minor)); IR (film): $\tilde{\nu}_{\max }=3074$ (w), 3001 (w), 2932 (m), 2862 (w), 2836 (m), 1682 (s), 1640 (m), 1609 (s), 1496 (m), 1464 (m), 1422 (s), 1358 (m), 1322 (m), 1277 (s), 1249 (s), 1200 (m), 1173 (m), 1036 (m), 997 (w), 915 (m), $877(\mathrm{~m}), 817(\mathrm{w}), 713 \mathrm{~cm}^{-1}(\mathrm{w}) ;{ }^{1} \mathrm{H}$ NMR (400 MHz, CDCl $): \delta=7.51(\mathrm{~d}, J=2.8 \mathrm{~Hz}, 1 \mathrm{H}), 7.14(\mathrm{~d}, J=$ $8.4 \mathrm{~Hz}, 1 \mathrm{H}), 7.04$ (dd, $\left.J_{1}=8.4 \mathrm{~Hz}, J_{2}=2.8 \mathrm{~Hz}, 1 \mathrm{H}\right), 5.84(\mathrm{~m}, 1 \mathrm{H}), 5.14-5.04(\mathrm{~m}, 2 \mathrm{H}), 3.83$ (s, $\left.3 \mathrm{H}\right), 2.92$ $\left(\mathrm{dd}, J_{1}=7.2 \mathrm{~Hz}, J_{2}=4.8 \mathrm{~Hz}, 2 \mathrm{H}\right), 2.75(\mathrm{~m}, 1 \mathrm{H}), 2.53(\mathrm{~m}, 1 \mathrm{H}), 2.23(\mathrm{~m}, 2 \mathrm{H}), 1.84(\mathrm{~m}, 1 \mathrm{H}) ;{ }^{13} \mathrm{C}$ NMR $(100$ $\left.\mathrm{MHz}, \mathrm{CDCl}_{3}\right): \delta=199.7,158.5,136.9,136.5,133.5,130.2,121.8,117.1,109.5,55.7,47.2$, 34.3, 28.4, 28.0; Anal. Calcd. for $\mathrm{C}_{14} \mathrm{H}_{16} \mathrm{O}_{2}$ : C, 77.75; H, 7.46; Found: C, 77.94 ; H, 7.21.

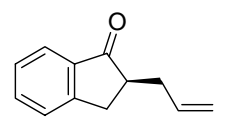

(+)-2-Allyl-1-indanone (28) ${ }^{12}$ : Colorless oil. $\mathrm{R}_{\mathrm{f}}=0.27$ (Diethyl ether/petroleum ether $1: 9$ ); $[\alpha]_{\mathrm{D}}{ }^{24}=+95.8$ (c = 4.0, $\mathrm{CH}_{2} \mathrm{Cl}_{2}, 81 \%$ ee); HPLC (Chiralcel ${ }^{\circledR}$ OB-H column; 90:10 Heptane / Isopropanol; flow rate $=1$ $\mathrm{mL} / \mathrm{min} ; \mathrm{t}_{1}=6.27$ min (minor), $\mathrm{t}_{2}=7.70$ min (major)); IR (film): $\tilde{\nu}_{\max }=3077(\mathrm{~m}), 2979(\mathrm{w}), 2919$ (s), 2848 (w), 1714 (s), 1641 (m), 1610 (s), 1588 (m), 1474 (m), 1464 (s), 1435 (s), 1326 (m), 1296 (s), 1274 (s), 1211 (m), 1183 (w), 1151 (m), 1094 (m), 998 (s), 917 (s), 791 (w), 754 (s), 725 (m), $657 \mathrm{~cm}^{-1}$ (m); ${ }^{1} \mathrm{H}$ NMR $\left(400 \mathrm{MHz}, \mathrm{CDCl}_{3}\right): \delta=7.76(\mathrm{~d}, J=7.6 \mathrm{~Hz}, 1 \mathrm{H}), 7.59\left(\mathrm{td}, J_{1}=7.6 \mathrm{~Hz}, J_{2}=1.0 \mathrm{~Hz}, 1 \mathrm{H}\right), 7.45(\mathrm{~d}, J=$ $8.0 \mathrm{~Hz}, 1 \mathrm{H}), 7.37(\mathrm{t}, J=7.6 \mathrm{~Hz}, 1 \mathrm{H}), 5.81(\mathrm{~m}, 1 \mathrm{H}), 5.16-5.02(\mathrm{~m}, 2 \mathrm{H}), 3.29$ (dd, $J_{1}=17.2 \mathrm{~Hz}, J_{2}=8.0 \mathrm{~Hz}$, $1 \mathrm{H}), 2.87\left(\mathrm{dd}, J_{1}=17.2 \mathrm{~Hz}, J_{2}=4.0 \mathrm{~Hz}, 1 \mathrm{H}\right), 2.82-2.66(\mathrm{~m}, 2 \mathrm{H}), 2.27(\mathrm{~m}, 1 \mathrm{H}) ;{ }^{13} \mathrm{C} \mathrm{NMR}(100 \mathrm{MHz}$, $\left.\mathrm{CDCl}_{3}\right): \delta=208.3,153.9,136.8,135.6,134.9,127.4,126.7,124.0,117.0,46.6,35.6,32.1$. 


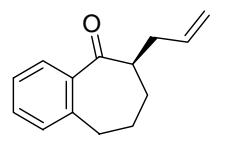

(+)-2-Allyl-1-benzosuberone (30) ${ }^{13}$ : Colorless oil. $\mathrm{R}_{\mathrm{f}}=0.45$ (Diethyl ether/petroleum ether 1:9); $[\alpha]_{\mathrm{D}}{ }^{23}=$ +70.43 (c = 1.56, $\mathrm{CH}_{2} \mathrm{Cl}_{2}$, 99\% ee); HPLC (Chiralcel ${ }^{\circledR}$ OB-H column; 2000:1 Heptane / Isopropanol; flow rate $=1 \mathrm{~mL} / \mathrm{min} ; \mathrm{t}_{1}=11.47 \mathrm{~min}$ (major), $\mathrm{t}_{2}=13.74 \mathrm{~min}$ (minor)); IR (film): $\tilde{\nu}_{\max }=3072(\mathrm{~m}), 2935$ (s), 2863 (m), 1682 (s), 1641 (m), 1599 (m), 1448 (m), 1375 (w), 1277 (m), 1246 (m), 1201 (m), 996 (w), 967 (m), 914 (s), $782(\mathrm{w}), 763(\mathrm{~m}), 737 \mathrm{~cm}^{-1}(\mathrm{~s}) ;{ }^{1} \mathrm{H}$ NMR (400 MHz, $\left.\mathrm{CDCl}_{3}\right): \delta=7.64$ (dd, $J_{1}=7.6 \mathrm{~Hz}, J_{2}=$ $1.0 \mathrm{~Hz}, 1 \mathrm{H}), 7.37$ (td, $\left.J_{1}=7.6 \mathrm{~Hz}, J_{2}=1.0 \mathrm{~Hz}, 1 \mathrm{H}\right), 7.27$ (t, $\left.J=7.6 \mathrm{~Hz}, 1 \mathrm{H}\right), 7.21$ (d, $\left.J=7.6 \mathrm{~Hz}, 1 \mathrm{H}\right), 5.77$ (m, 1H), 5.08 - 4.97 (m, 2H), 2.94 (m, 3H), 2.68 (m, 1H), 2.23 (p, J = 7.2 Hz, 1H), 2.06 (m, 1H), 1.96 (m, $1 \mathrm{H}), 1.69(\mathrm{~m}, 1 \mathrm{H}), 1.59(\mathrm{~m}, 1 \mathrm{H}) ;{ }^{13} \mathrm{C}$ NMR $\left(100 \mathrm{MHz}, \mathrm{CDCl}_{3}\right): \delta=206.9,141.9,140.0,136.4,131.3$, 129.8, 128.3, 126.4, 116.5, 49.4, 35.4, 33.6, 29.7, 25.4.

(1) Perrin, D. D. Armarego, W. L. F. Purification of Laboratory Chemicals; Pergamon Press: New York, 1988.

(2) Ukai, T.; Kawazura, H.; Ishii, Y.; Bonnet, J. J.; Ibers, J. A. J. Organometallic Chem. 1974, 65, 253-266.

(3) Trost, B. M.; Van Vranken, D. L.; Bingel, C. J. Am. Chem. Soc. 1992, 114, 9327-9343.

(4) Trost, B. M.; Bunt, R. C.; Lemoine, R. C.; Calkins, T. L. J. Am. Chem. Soc. 2000, 122, 5968-5976.

(5) Tsuji, J.; Minami, I.; Shimizu, I. Tetrohedron Lett. 1983, 24, 4713-4714.

(6) Imai, M.; Hagihara, A.; Kawasaki, H.; Manabe, K.; Koga, K. Tetrahedron 2000, 56, 179-185.

(7) Diez-Barra, E.; Medrino, S.; Sanchez-Verdu, P.; Torres, J. Tetrahedron 1997, 53, 11437-11448.

(8) Meyers, A. I.; Williams, D. R.; Erickson, G. W.; Whites, S.; Druelinger, M. J. Am. Chem. Soc. 1981, 103, 3081-3087.

(9) Trost, B. M.; Schroeder, G. M. J. Am. Chem. Soc. 1999, 121, 6759-6760.

(10) Schroeder, G. M. Palladium Catalyzed Asymmetric Allylic Alkylation (AAA) of Prochiral Nucleophiles: Efforts toward the Synthesis of Erinacine A. Ph.D. Dissertation, Stanford University: Stanford, 2002.

(11) Cid, J. M.; Romera, J. L.; Trabanco, A. A. Tetrahedron Lett. 2004, 45, 1133-1136.

(12) Tsuda, T.; Okada, M.; Nishi, S.; Saegusa, T. J. Org. Chem. 1986, 51, 421-426.

(13) Davies, H. J.; Main, B. G. J. Chem. Soc. C-Organic 1970, 237. 


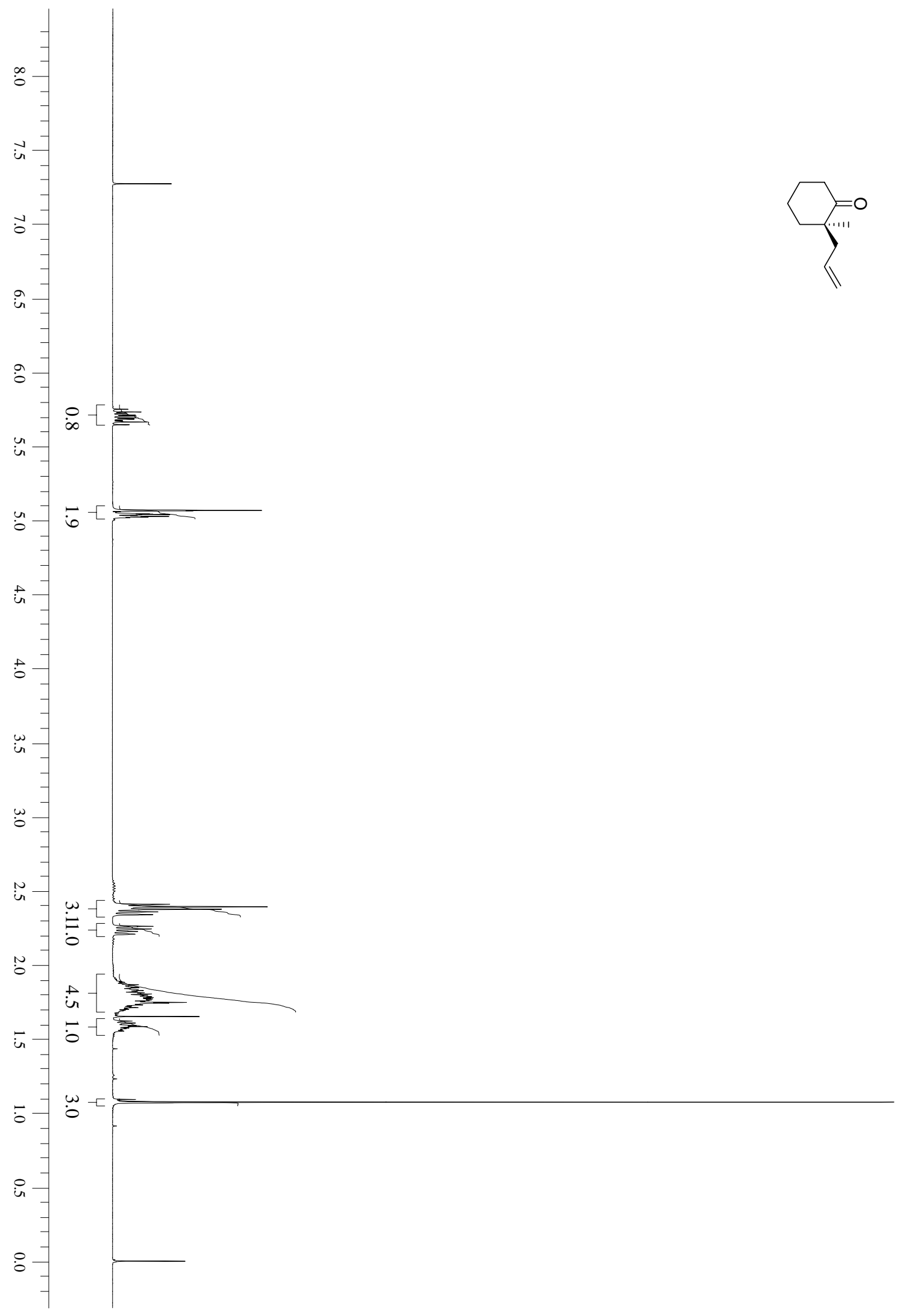



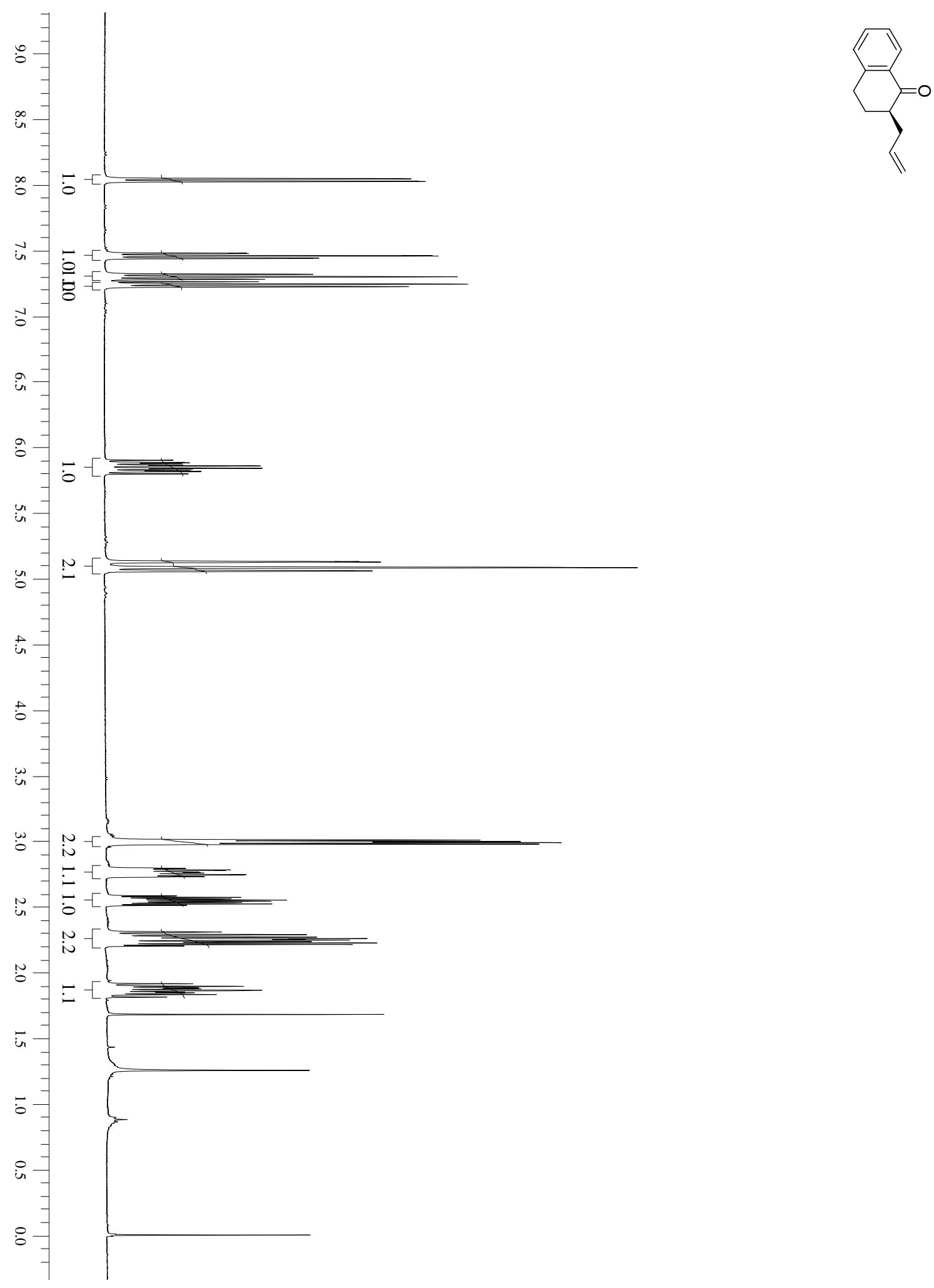


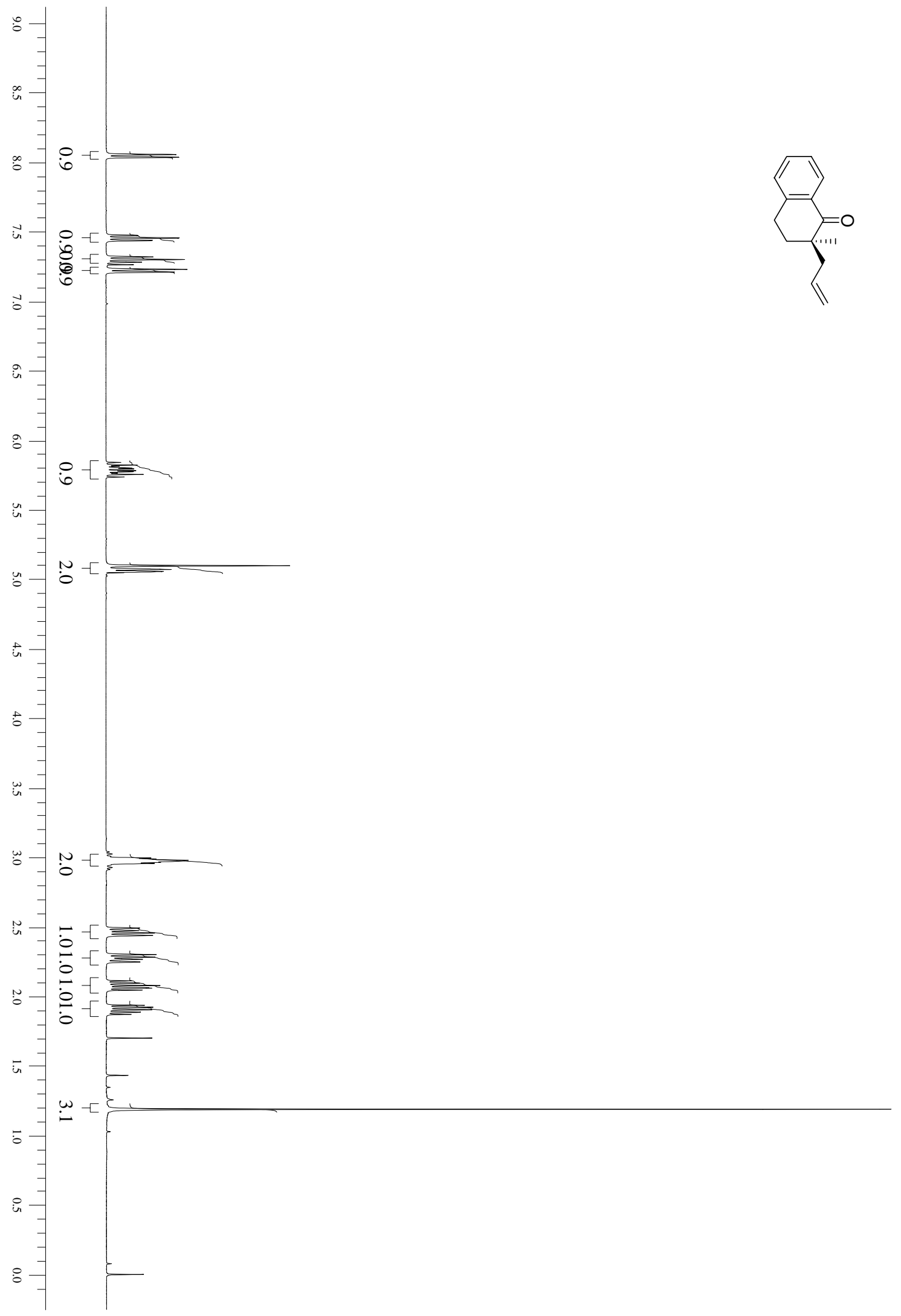




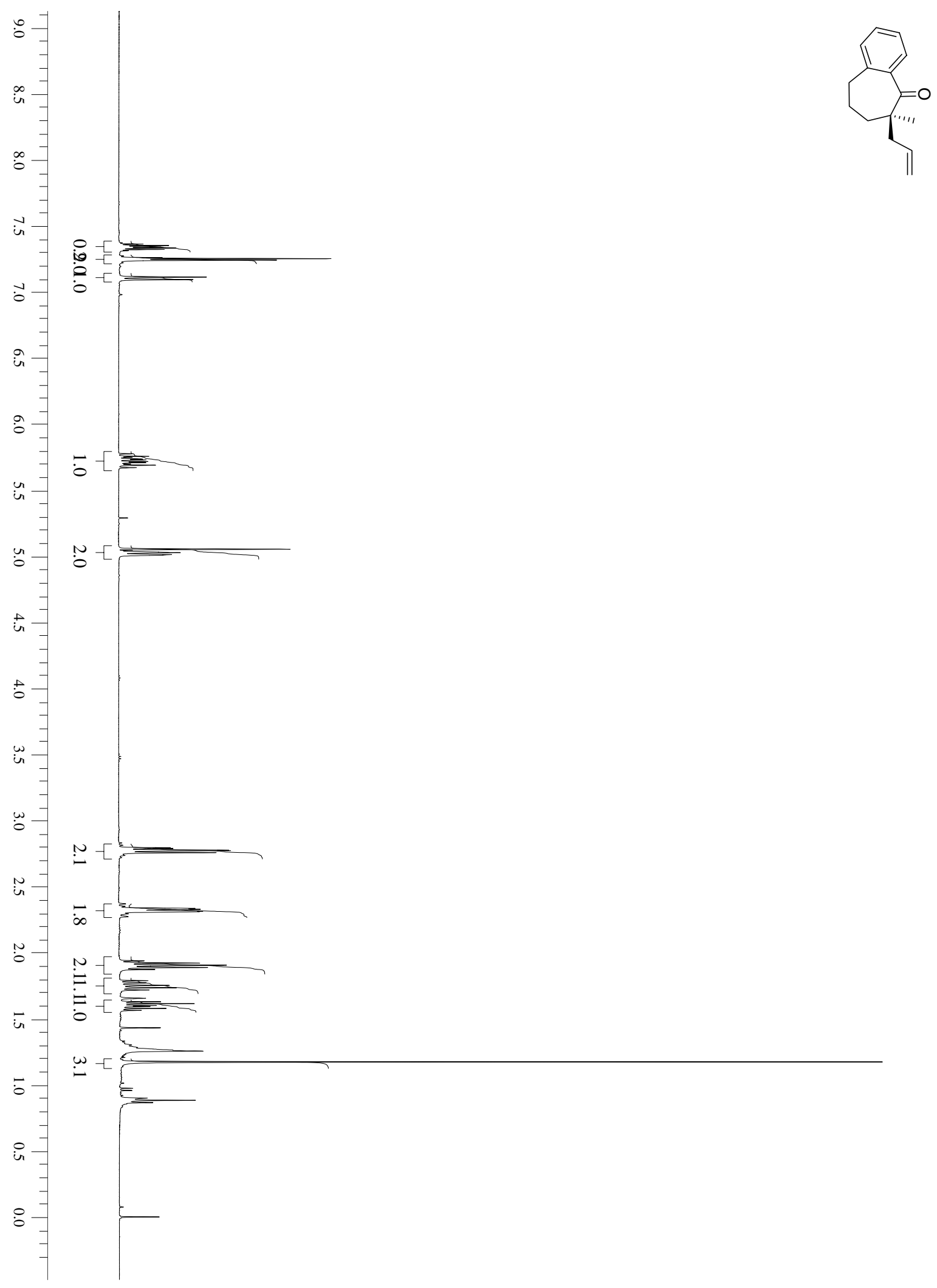




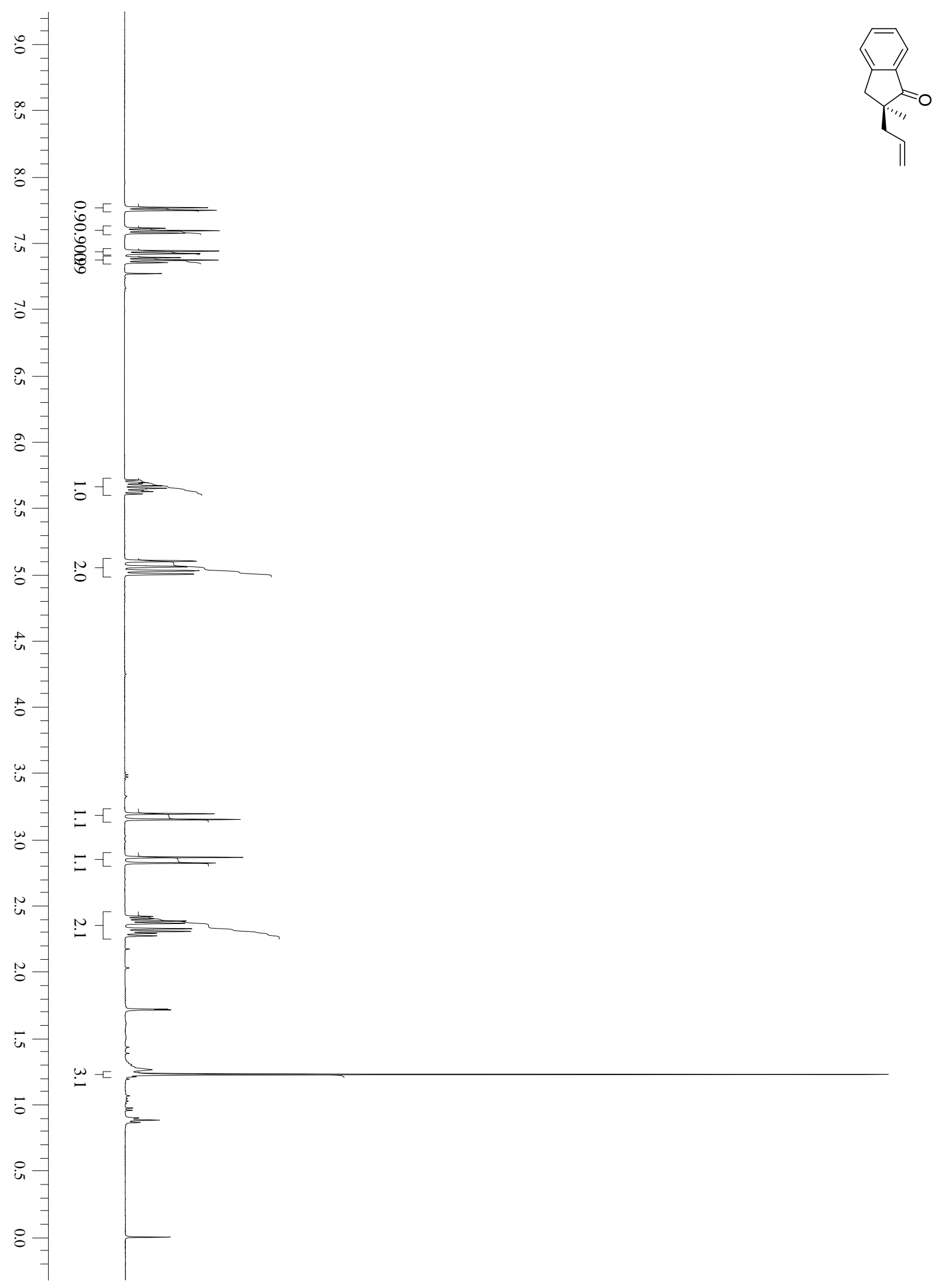




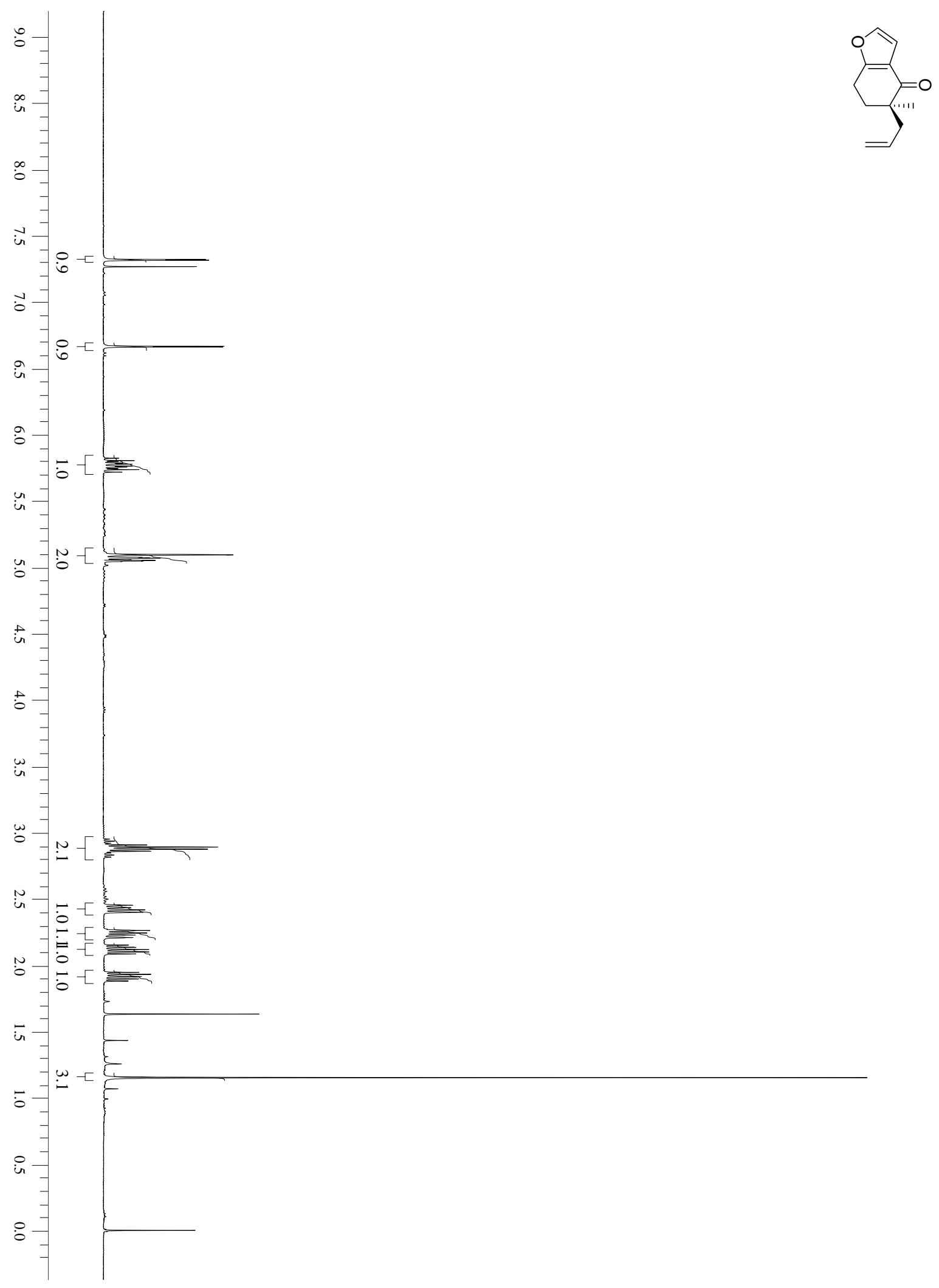




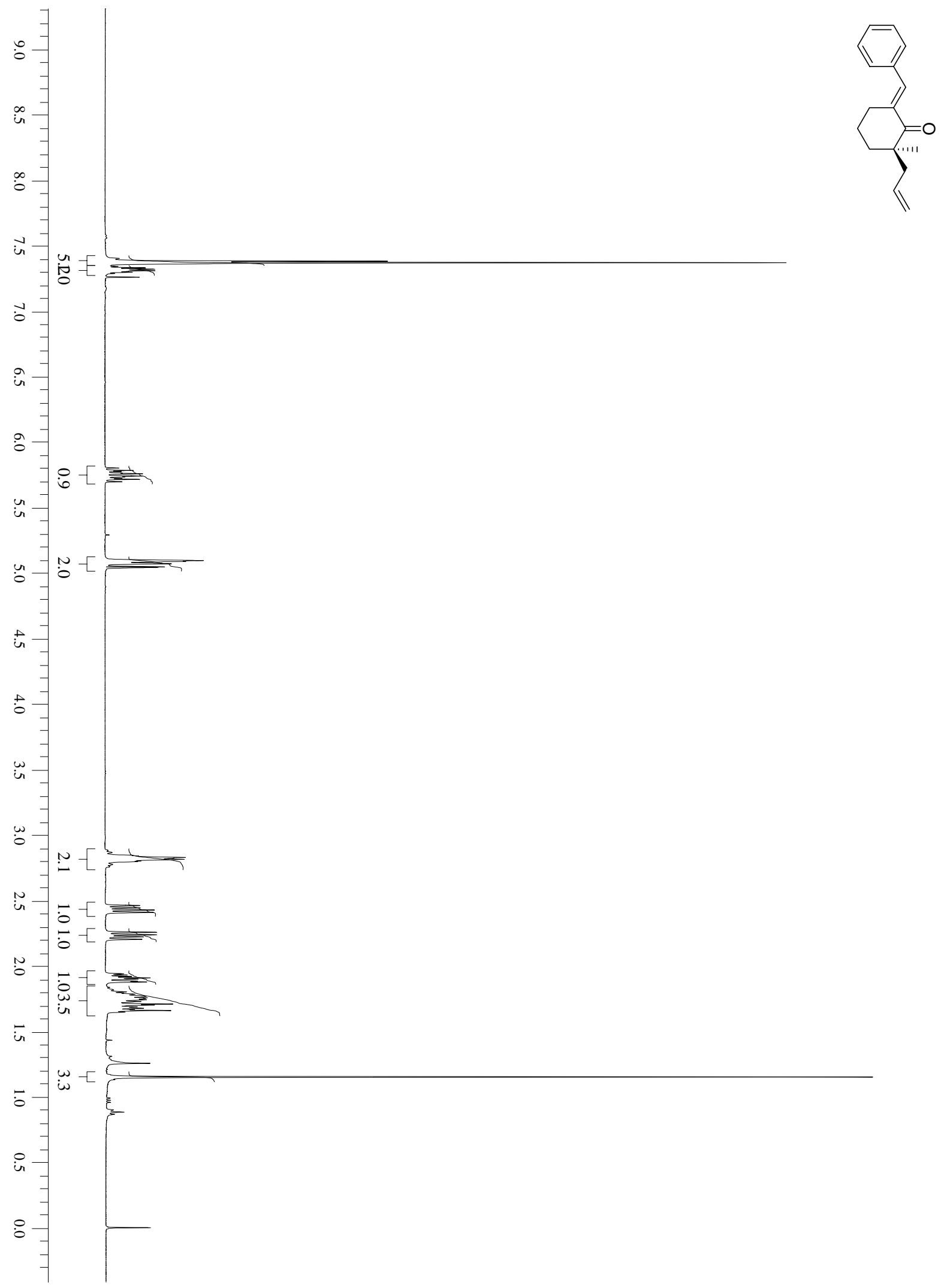



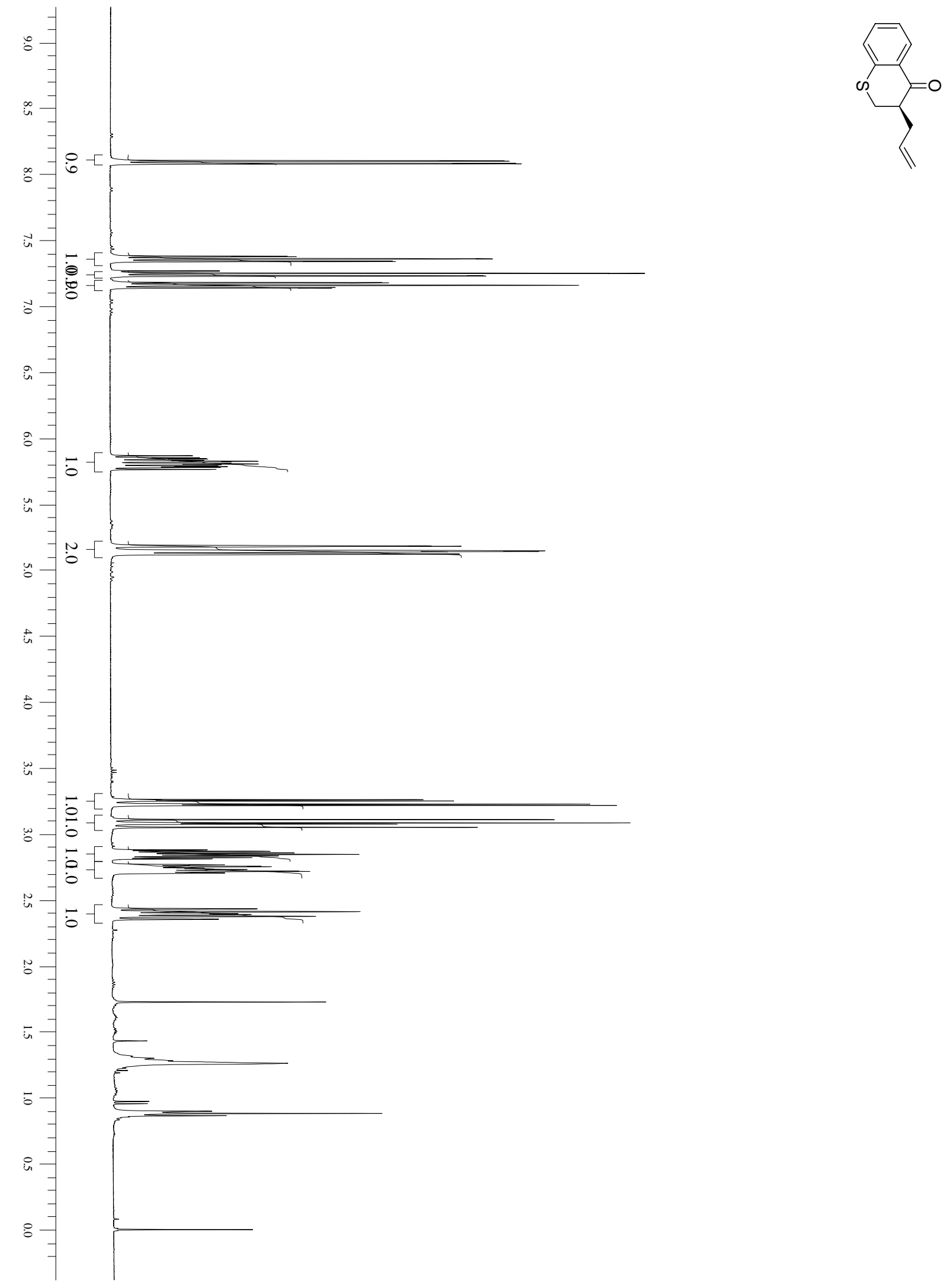


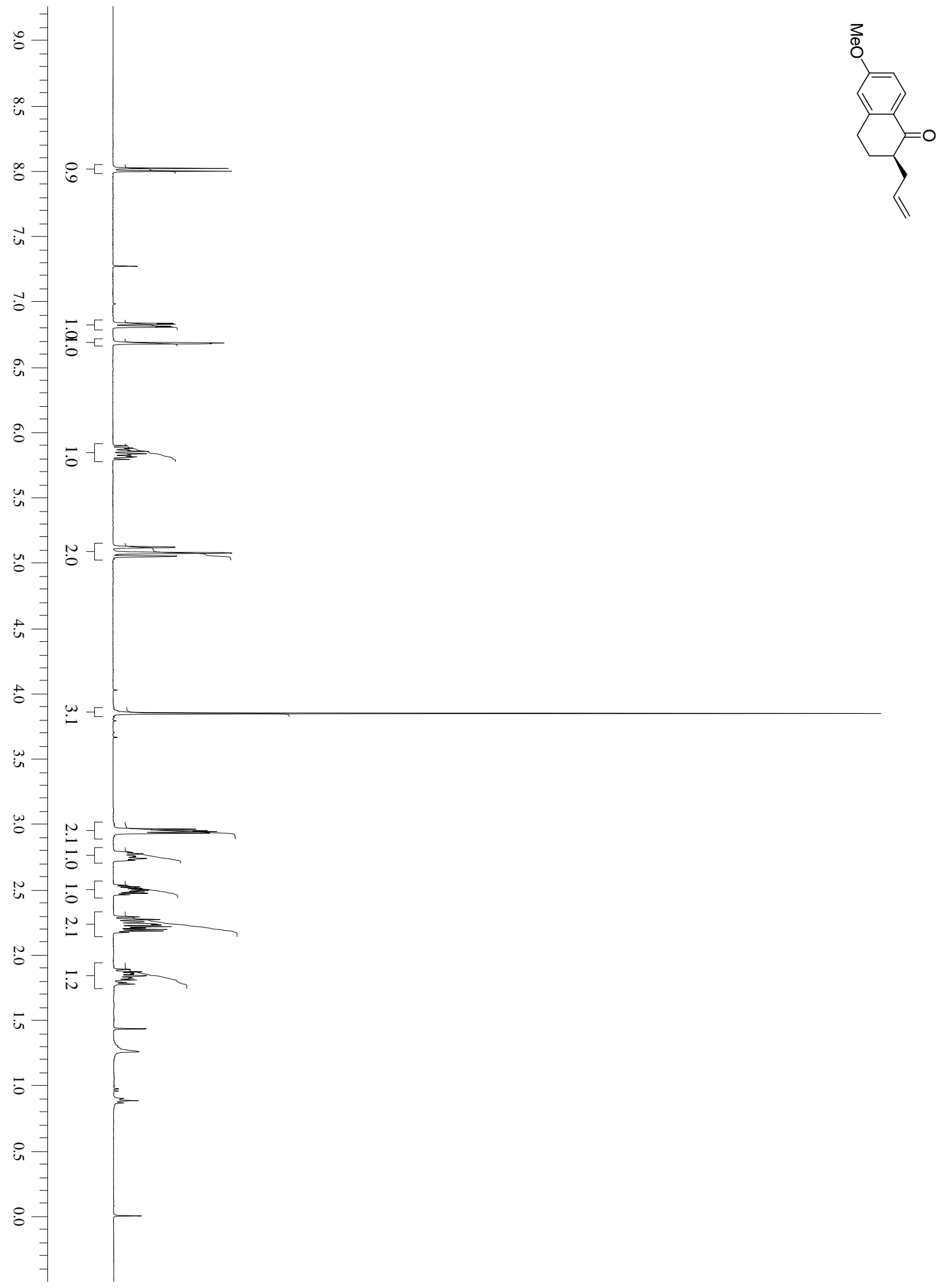




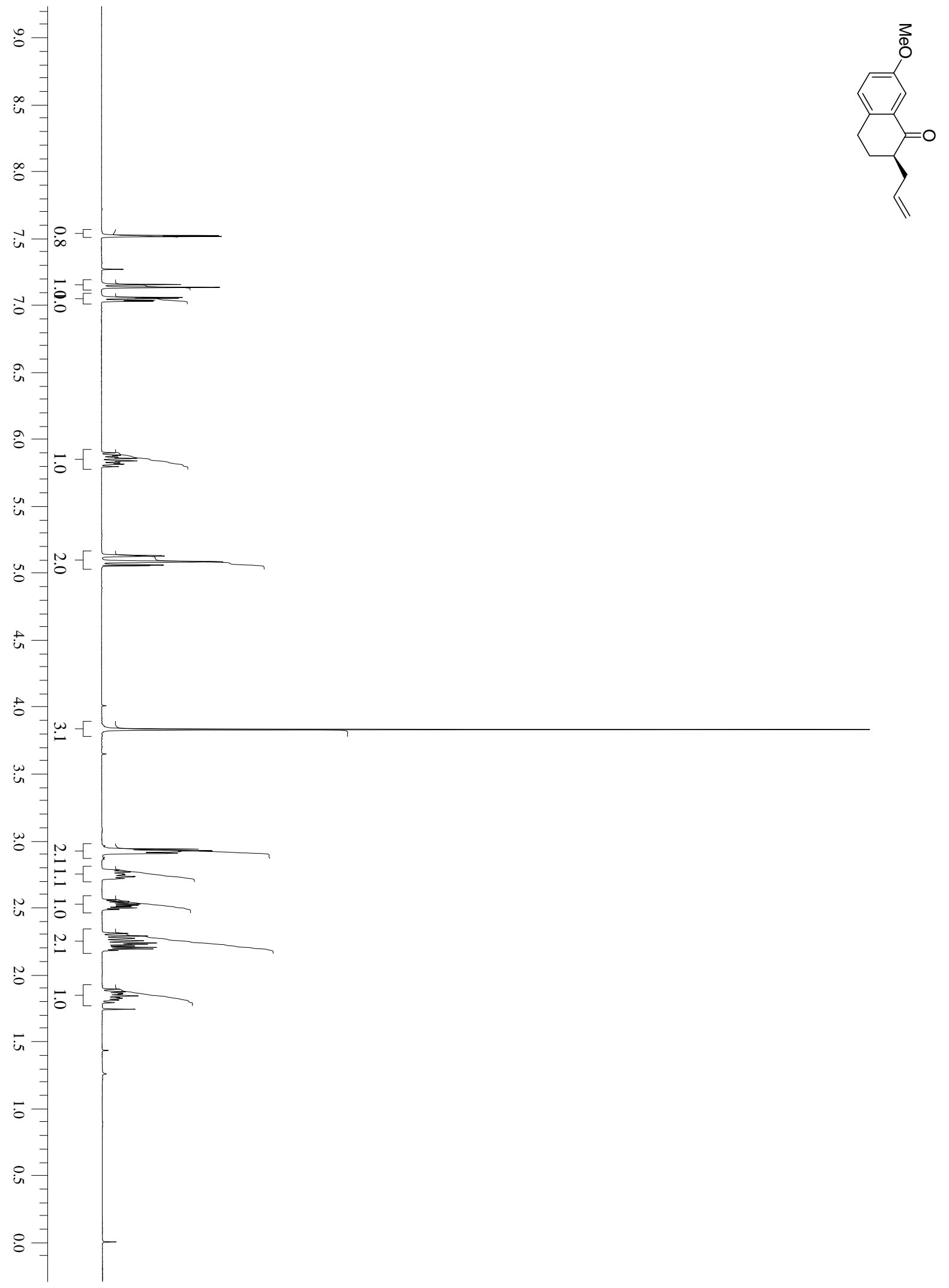



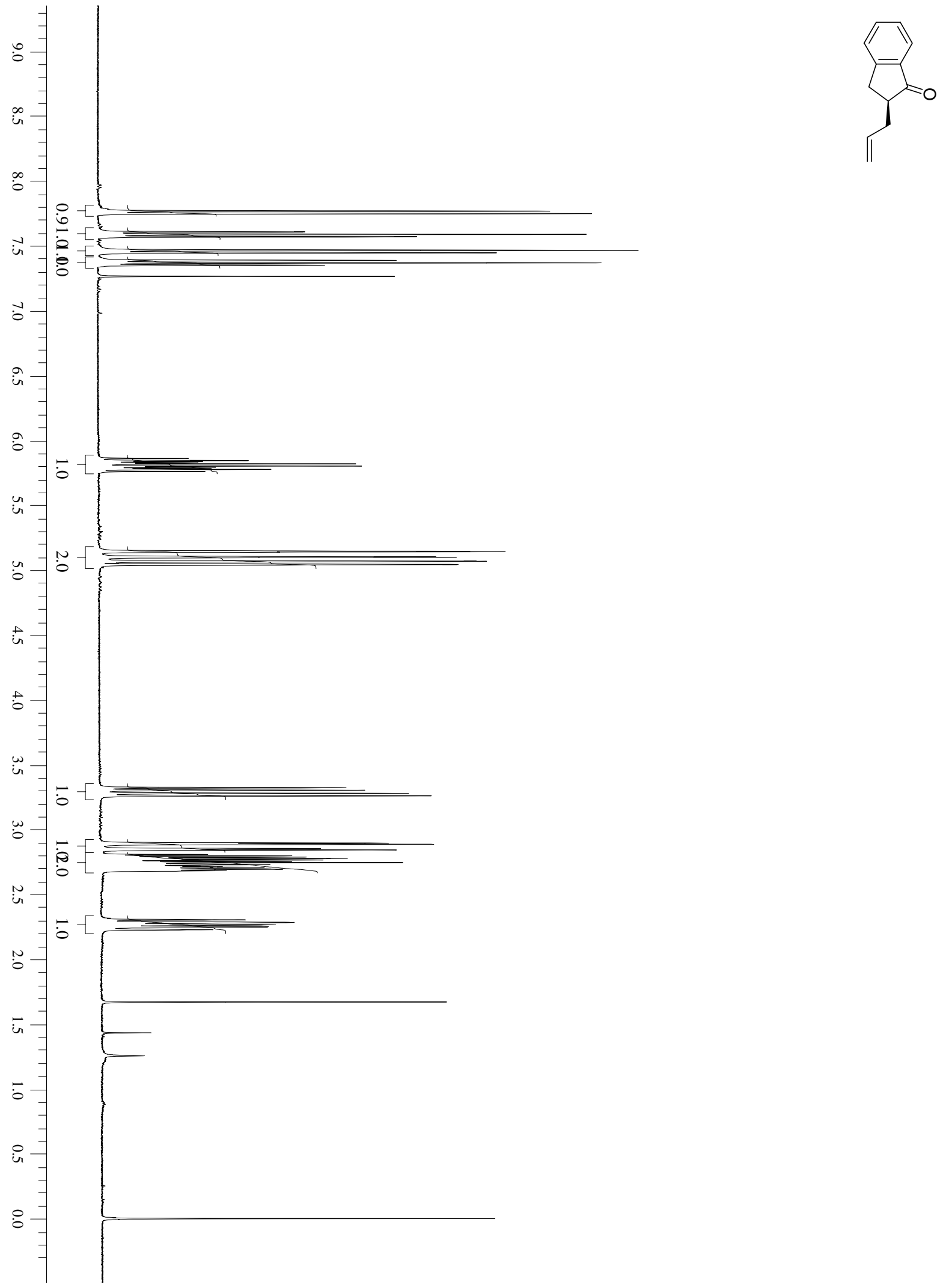

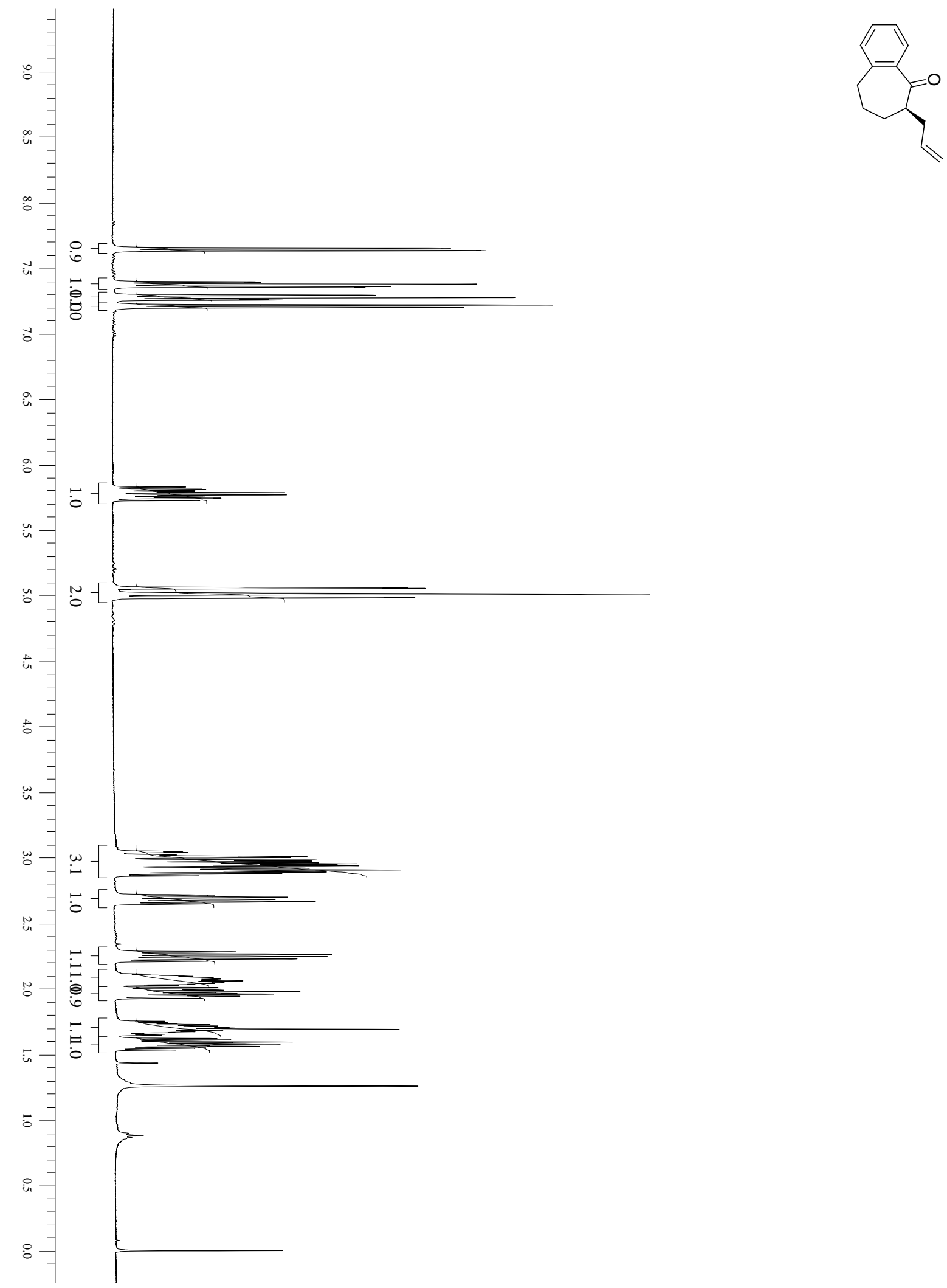


\section{Crossover Experiment:}<smiles>C=CCOC(=O)OC1=CCCc2cc(OC)ccc21</smiles><smiles>C/C=C\COC(=O)OC1=CCCc2ccc(OC)cc21</smiles>

31<smiles>C=CCC1CCc2cc(OC)ccc2C1=O</smiles>

24 $2.5 \% \mathrm{Pd}_{2}(\mathrm{dba})_{3} \mathrm{CHCl}_{3}$ 5.5\% L4, Dioxane, r.t.<smiles>C=CCC1CCc2ccc(OC)cc2C1=O</smiles>

26<smiles>C=CC(C)C1CCc2cc(OC)ccc2C1=O</smiles><smiles>C/C=C\CC1CCc2ccc(OC)cc2C1=O</smiles><smiles>C=CC(C)C1CCc2ccc(OC)cc2C1=O</smiles>
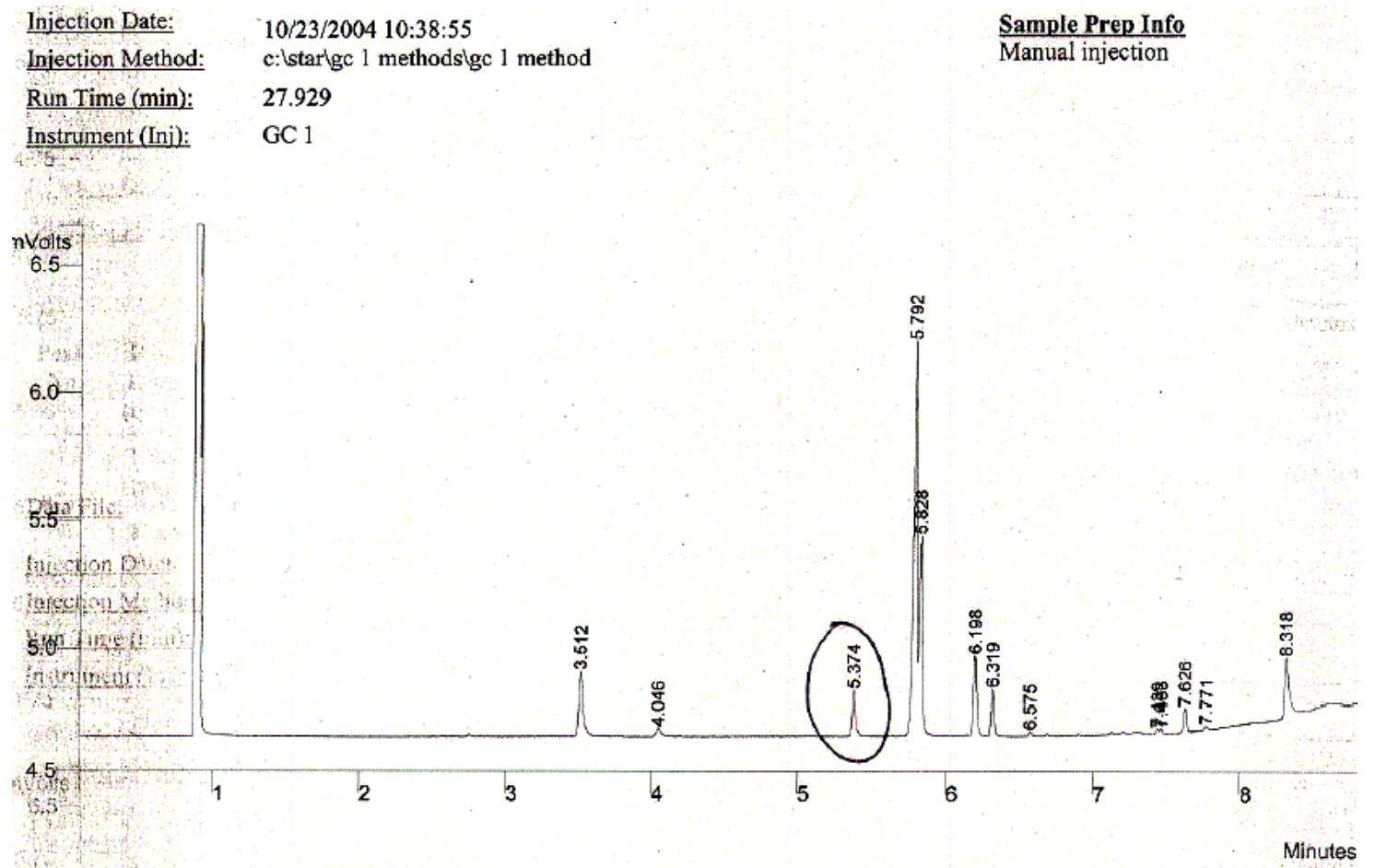

\begin{tabular}{|c|c|c|c|c|}
\hline $\begin{array}{c}\text { Peak } \\
\text { No }\end{array}$ & $\begin{array}{l}\text { Ret. } \\
\text { Time } \\
\text { (min) }\end{array}$ & Peak Area & $\begin{array}{c}\text { Width } \\
1 / 2 \\
\text { (sec) }\end{array}$ & $\begin{array}{c}\text { Percent } \\
\text { Area }\end{array}$ \\
\hline 1 & 3.512 & 526 & 1.8 & 8.82 \\
\hline 2 & 4.046 & 76 & 2.1 & 1.27 \\
\hline $\operatorname{sen} 3$ & 5.374 & 277 & 1.4 & 4.64 \\
\hline 4 & 5.792 & 2610 & 1.5 & 43.79 \\
\hline 5 & 5.828 & 1063 & 1.3 & 17.84 \\
\hline 6 & 6.198 & 476 & 1.4 & 7.98 \\
\hline 02 & 6.319 & 253 & 1.2 & 4.25 \\
\hline 148 & 6.575 & 30 & 1.4 & 0.50 \\
\hline 9 & 7.439 & 17 & 1.1 & 0.28 \\
\hline 10 & 7.463 & 25 & 1.2 & 0.42 \\
\hline 4.11 & 7.626 & 117 & 1.2 & 1.96 \\
\hline 12 & 7.771 & 22 & 1,5 & 0.37 \\
\hline 13 & 8.318 & 470 & 1.8 & 7.88 \\
\hline Peak & Retio & 5962 & & 100.00 \\
\hline
\end{tabular}

GC\#1 Method C

Method Notes

No Method Notes 


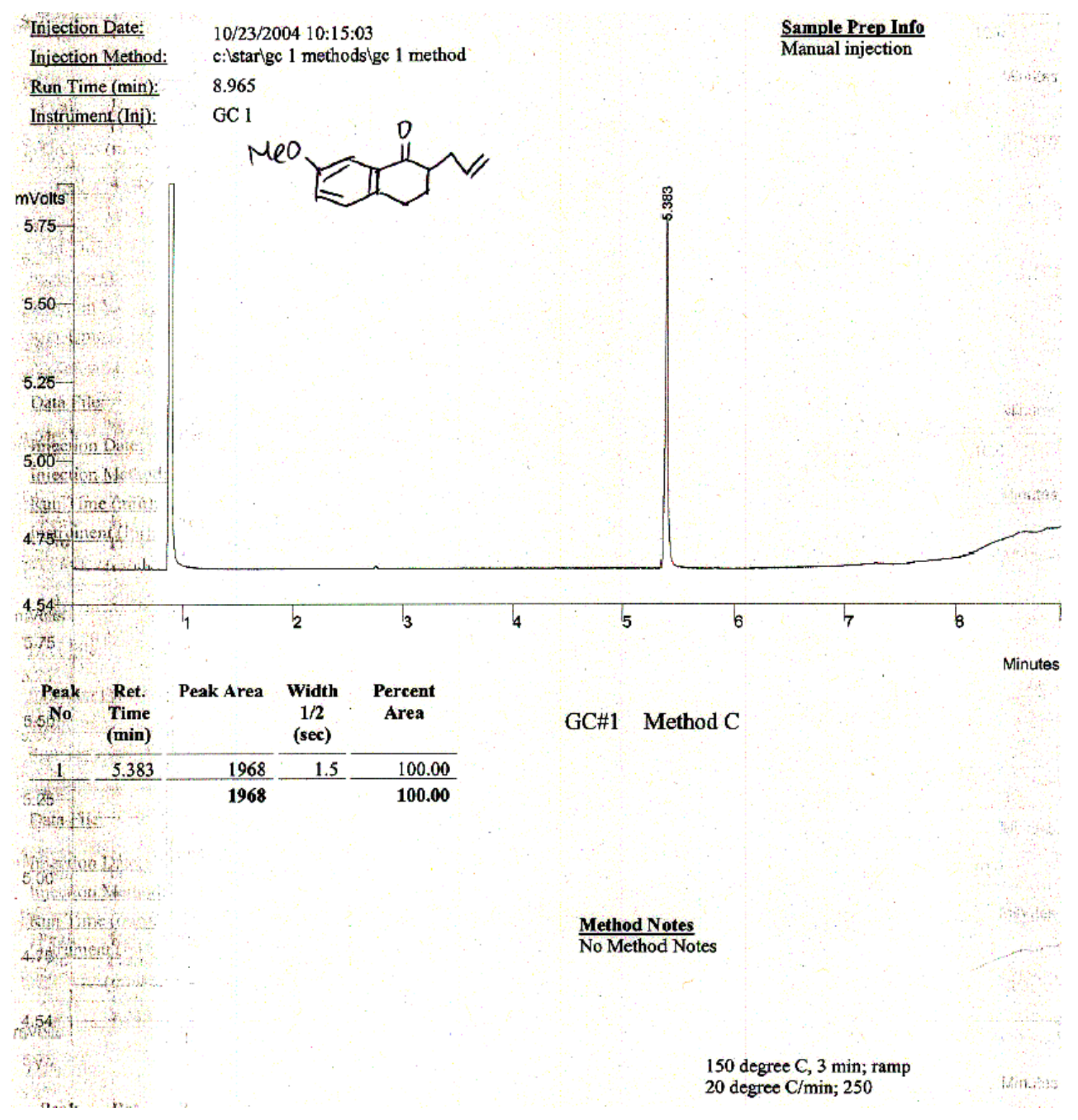




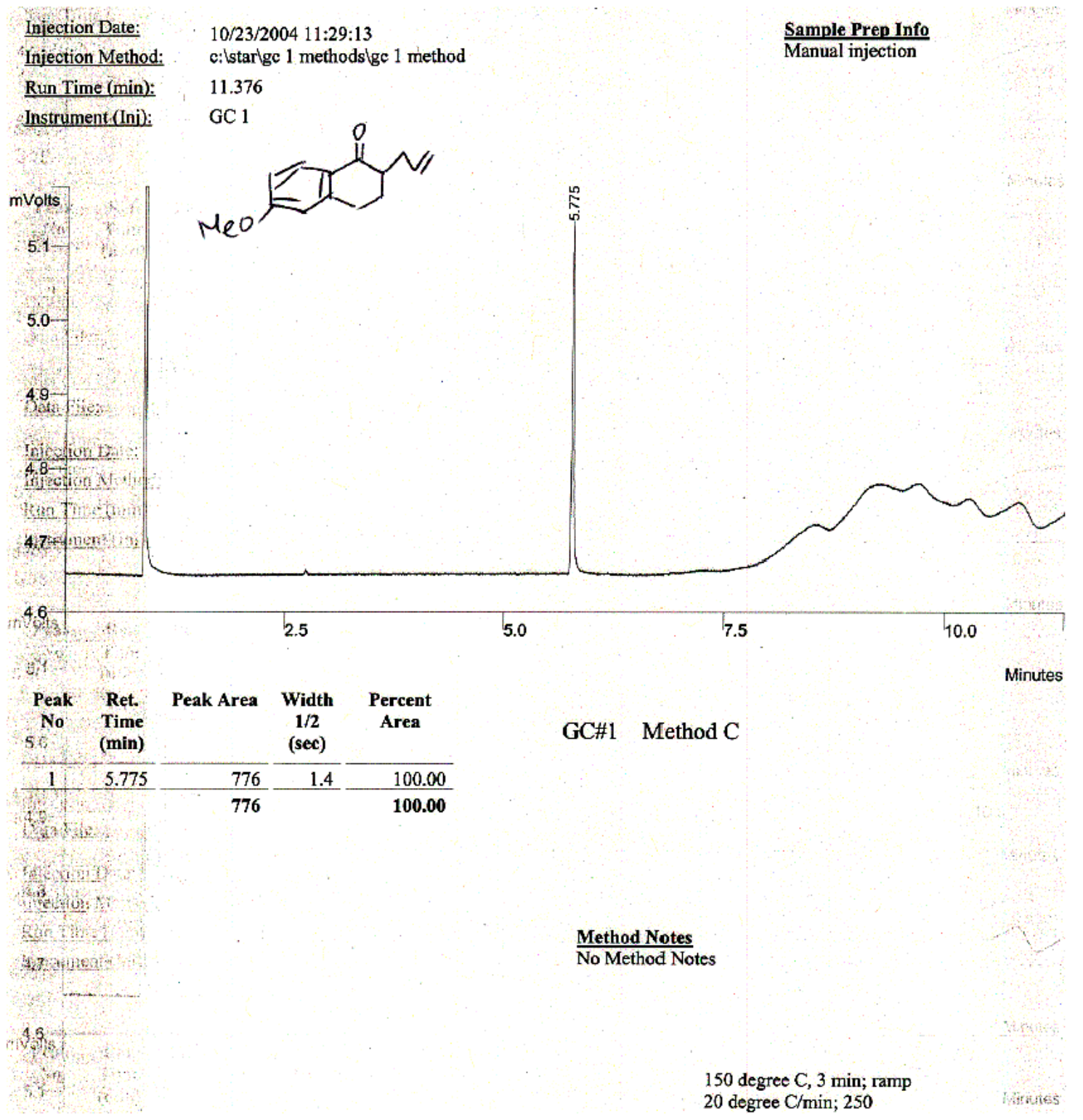


<smiles>C/C=C\COC(=O)OC1=CCCc2ccc(OC)cc21</smiles>

31<smiles>C=CC(C)C1CCc2ccc(OC)cc2C1=O</smiles>

1:1 mixture by ${ }^{1} \mathrm{HNMR}$, unseparable on TLC

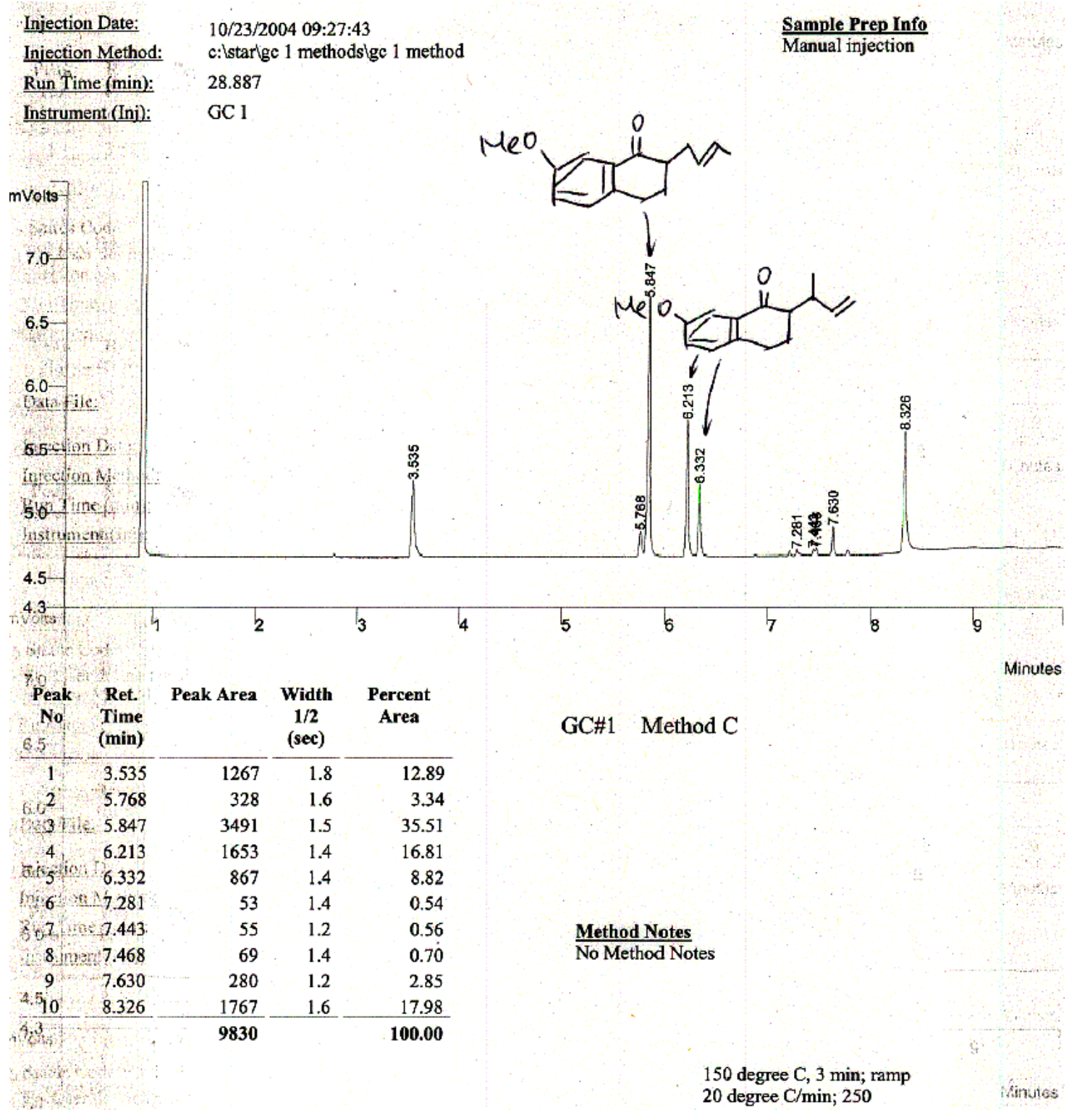




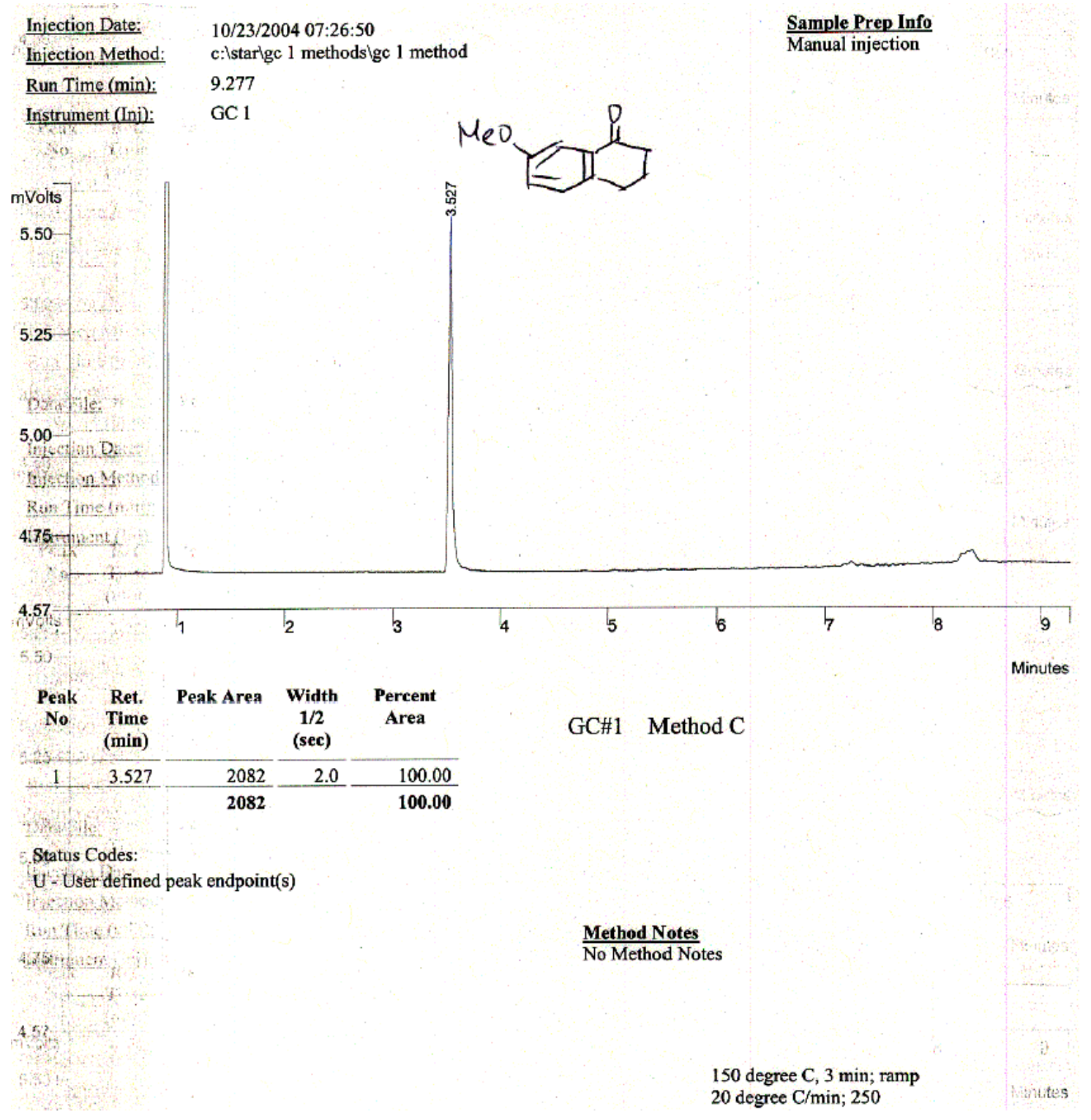




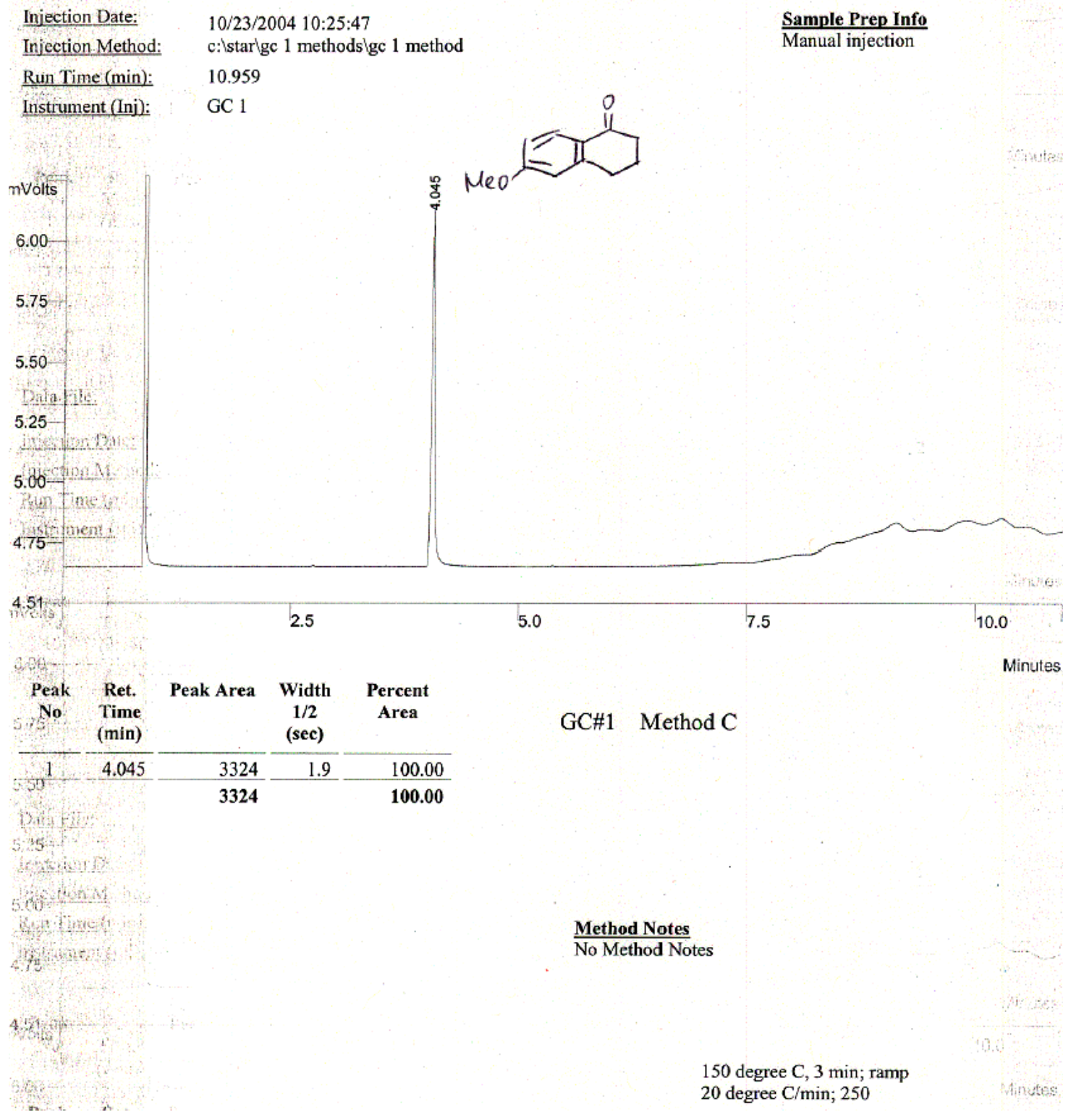




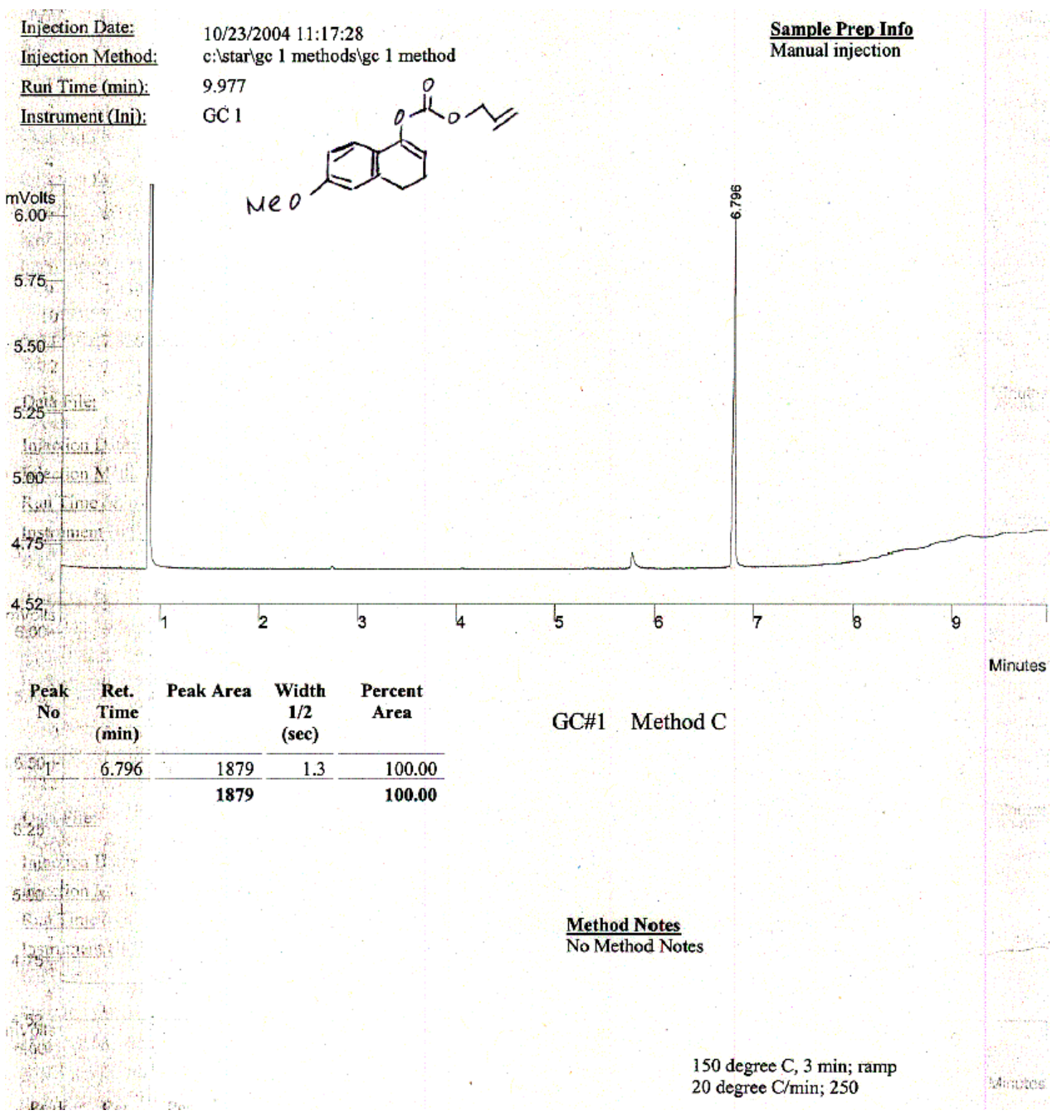




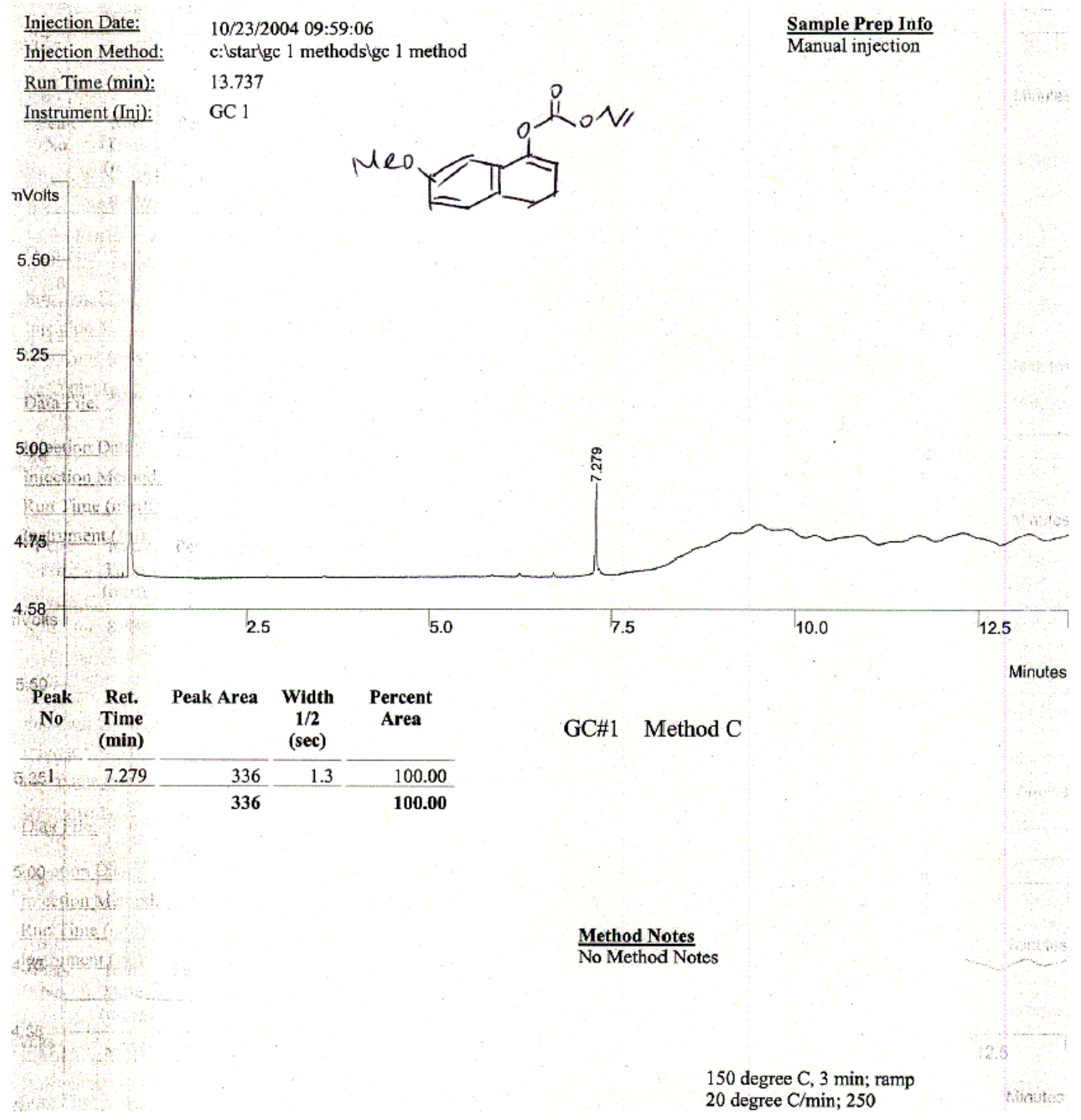




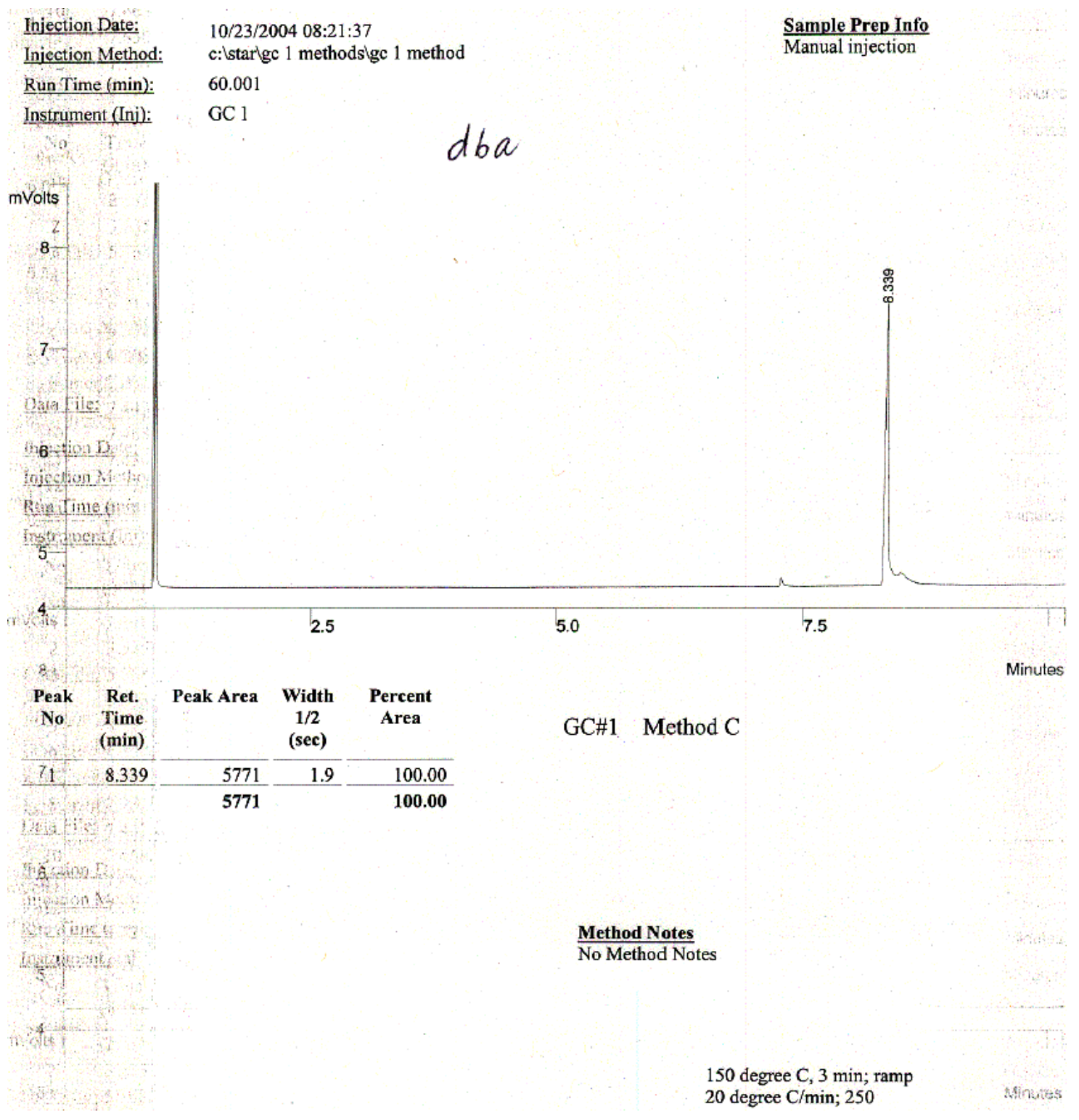

